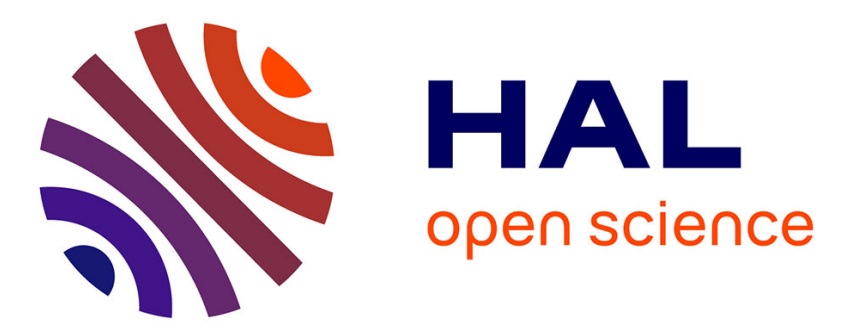

\title{
The cross-motion invariant group and its application to kinematics
}

\author{
Bruno Vilhena Adorno, Philippe Fraisse
}

\section{To cite this version:}

Bruno Vilhena Adorno, Philippe Fraisse. The cross-motion invariant group and its application to kinematics. IMA Journal of Mathematical Control and Information, 2017, 34 (4), pp.1359-1378. 10.1093/imamci/dnw032 . lirmm-01396641

\section{HAL Id: lirmm-01396641 https://hal-lirmm.ccsd.cnrs.fr/lirmm-01396641}

Submitted on 14 Nov 2016

HAL is a multi-disciplinary open access archive for the deposit and dissemination of scientific research documents, whether they are published or not. The documents may come from teaching and research institutions in France or abroad, or from public or private research centers.
L'archive ouverte pluridisciplinaire HAL, est destinée au dépôt et à la diffusion de documents scientifiques de niveau recherche, publiés ou non, émanant des établissements d'enseignement et de recherche français ou étrangers, des laboratoires publics ou privés. 
IMA Journal of Mathematical Control and Information (2016) 00, 1-20

doi: 10.1093/imamci/dnw032

\title{
The cross-motion invariant group and its application to kinematics
}

\author{
BRUNO VILHENA AdORNO* \\ Departamento de Engenharia Elétrica, Universidade Federal de Minas Gerais, \\ Av. Antônio Carlos 6627, CEP 31270-010, Belo Horizonte, Minas Gerais, Brazil \\ *Corresponding author: adorno@ufmg.br \\ AND \\ PHILIPPE FRAISSE \\ Département de Robotique, Université Montpellier 2, LIRMM UMR 5506,CNRS, INRIA, DEMAR \\ Team - CC 477161 rue Ada, 34392 Montpellier Cedex 5, France \\ [Received on 31 January 2016; revised on 10 April 2016; accepted on 19 May 2016]

\begin{abstract}
This article presents the cross-motion invariant group-CMI(3) - whose group operation is defined over unit dual quaternions such that rigid motions are cross-motion invariant; that is, the resultant translation does not depend on rotation and vice-versa. We present the main properties of $C M I(3)$ and the differences between this group and the standard group $\operatorname{Spin}(3) \ltimes \mathbb{R}^{3}$ of unit dual quaternions, as well as the kinematic equations under a sequence of $C M I(3)$ operations. Two numerical examples are presented in order to illustrate the main characteristics of $C M I(3)$.
\end{abstract}

Keywords: kinematics; unit dual quaternion; rigid motion; cross-motion invariance.

\section{Introduction}

The study of rigid motions is a classic topic in robot kinematics and is covered by virtually all modern robotics textbooks (for example, see Murray et al., 1994; Craig, 2005; Spong et al., 2006; Siciliano \& Khatib, 2008; Siciliano et al., 2009). Its importance relies on the fact that rigid motions (or rigid transformations) play an important role in robot modelling and control (Spong et al., 2006), as well as in motion planning and high-level task definitions (Lana et al., 2015), as most robots are composed of rigid components serially or parallelly attached to each other, resulting in complex kinematic chains. The group that represents rigid motions in three dimensions is the Special Euclidean Group $S E(3)$ and it is well known that this group is basically the semi-direct product of $S O(3)$ and $\mathbb{R}^{3}$ (Selig, 2005); that is, given $\boldsymbol{R} \in S O(3)$ and $\boldsymbol{p} \in \mathbb{R}^{3}$ such that $\boldsymbol{X}=(\boldsymbol{R}, \boldsymbol{p}) \in S E(3)$, then

$$
\boldsymbol{X}_{1} \boldsymbol{X}_{2}=\left(\boldsymbol{R}_{1}, \boldsymbol{p}_{1}\right)\left(\boldsymbol{R}_{2}, \boldsymbol{p}_{2}\right)=\left(\boldsymbol{R}_{1} \boldsymbol{R}_{2}, \boldsymbol{p}_{1}+\boldsymbol{R}_{1} \boldsymbol{p}_{2}\right) .
$$

Elements of SE(3) are usually written in the form of homogeneous transformation matrices (HTM); that is,

$$
\boldsymbol{X}=\left[\begin{array}{cc}
\boldsymbol{R} & \boldsymbol{p} \\
\mathbf{0}_{1 \times 3} & 1
\end{array}\right]
$$

where $\mathbf{0}_{m \times n}$ is the zero matrix with $m$ rows and $n$ columns, such that

$$
\left[\begin{array}{cc}
\boldsymbol{R}_{1} & \boldsymbol{p}_{1} \\
\mathbf{0}_{1 \times 3} & 1
\end{array}\right]\left[\begin{array}{cc}
\boldsymbol{R}_{2} & \boldsymbol{p}_{2} \\
\mathbf{0}_{1 \times 3} & 1
\end{array}\right]=\left[\begin{array}{cc}
\boldsymbol{R}_{1} \boldsymbol{R}_{2} & \boldsymbol{p}_{1}+\boldsymbol{R}_{1} \boldsymbol{p}_{2} \\
\mathbf{0}_{1 \times 3} & 1
\end{array}\right] .
$$


An alternative to the group $S E$ (3) in the representation of rigid transformations is the group $\operatorname{Spin}(3) \ltimes$ $\mathbb{R}^{3}$ of unit dual quaternions, which double covers $S E(3)$ (McCarthy, 1990; Selig, 2005). The advantage of using unit dual quaternions is that they are more compact than HTM, as the former has eight elements, whereas the latter has sixteen. In addition, dual quaternions have strong algebraic properties and can be used to represent rigid motions, twists, wrenches and several geometrical primitives-e.g. Plücker lines and planes - in a very straightforward way (Daniilidis, 1999; Radavelli et al., 2014). Moreover, it is easy to extract geometric parameters from a given unit dual quaternion (translation, axis of rotation and angle of rotation) and dual quaternions multiplications are less expensive than HTM multiplications (Adorno, 2011, p. 42). Also, unit dual quaternions do not have representational singularities (although this feature is also present in HTM) and are easily mapped into a vector structure, which can be particularly convenient when controlling a robot, as there is no need to extract parameters from the dual quaternion to perform such task (Adorno et al., 2010). Thanks to the aforementioned advantages, unit dual quaternions will be used throughout the article in order to represent rigid motions.

Let us introduce some facts about quaternions and dual quaternions. Let $\hat{\imath}, \hat{\jmath}, \hat{k}$ be the three imaginary units such that $\hat{\imath}^{2}=\hat{\jmath}^{2}=\hat{k}^{2}=\hat{\imath} \hat{\jmath} \hat{k}=-1$. The set of quaternions naturally extends the set of complex numbers and is defined as

$$
\mathbb{H} \triangleq\{a+b \hat{\imath}+c \hat{\jmath}+d \hat{k}: a, b, c, d \in \mathbb{R}\}
$$

whereas the set of pure quaternions is composed of all quaternions with real component equal to zero,

$$
\mathbb{H}_{p} \triangleq\{a \hat{\imath}+b \hat{\jmath}+c \hat{k}: a, b, c \in \mathbb{R}\}
$$

It is important to note that $\mathbb{H}_{p}$ is isomorphic to $\mathbb{R}^{3}$ under the addition operation; that is, $\left(\mathbb{R}^{3},+\right) \cong\left(\mathbb{H}_{p},+\right)$. The conjugate of a quaternion is analogous to the complex conjugate: given $\boldsymbol{h}=a+b \hat{\imath}+c \hat{\jmath}+d \hat{k}$, its conjugate is $\boldsymbol{h}^{*} \triangleq a-(b \hat{\imath}+c \hat{\jmath}+d \hat{k})$. In addition, the quaternion norm is defined as $\|\boldsymbol{h}\| \triangleq \sqrt{\boldsymbol{h} \boldsymbol{h}^{*}}$.

A unit quaternion (i.e. a quaternion with unit norm) $\boldsymbol{r}=\cos (\phi / 2)+\sin (\phi / 2) \boldsymbol{n}$ represents the rotation angle $\phi$ around the rotation axis $\boldsymbol{n}=n_{x} \hat{\imath}+n_{y} \hat{\jmath}+n_{z} \hat{k}$ and form the group Spin(3) (Kuipers, 2002; Selig, 2005). The identity element of $\operatorname{Spin}(3)$ is the real number 1 , since $1 \boldsymbol{r}=\boldsymbol{r} 1=\boldsymbol{r}$ and the group inverse is the conjugate $\boldsymbol{r}^{*} \triangleq \cos (\phi / 2)-\sin (\phi / 2) \boldsymbol{n}$, such that $\boldsymbol{r} \boldsymbol{r}^{*}=\boldsymbol{r}^{*} \boldsymbol{r}=1$. Thanks to the isomorphism between $\mathbb{R}^{3}$ and $\mathbb{H}_{p}$, pure quaternions represent translations and points in three dimensions, hence $\boldsymbol{p}=x \hat{\imath}+y \hat{\jmath}+z \hat{k}$ represents the point with coordinates $(x, y, z)$.

Elements of the group $\operatorname{Spin}(3) \ltimes \mathbb{R}^{3}$ behave analogously to elements of $S E(3)$, that is, given $\underline{x}=(\boldsymbol{r}, \boldsymbol{p}) \in \operatorname{Spin}(3) \ltimes \mathbb{R}^{3}$, then

$$
\underline{\boldsymbol{x}}_{1} \underline{\boldsymbol{x}}_{2}=\left(\boldsymbol{r}_{1}, \boldsymbol{p}_{1}\right)\left(\boldsymbol{r}_{2}, \boldsymbol{p}_{2}\right)=\left(\boldsymbol{r}_{1} \boldsymbol{r}_{2}, \boldsymbol{p}_{1}+\operatorname{Ad}\left(\boldsymbol{r}_{1}\right) \boldsymbol{p}_{2}\right),
$$

where $\operatorname{Ad}(\boldsymbol{r}) \boldsymbol{p} \triangleq \boldsymbol{r} \boldsymbol{p} \boldsymbol{r}^{*}$ represents the point $\boldsymbol{p}$ rotated by $\boldsymbol{r}$ (it is important to recall that (2) is equivalent to (1)). However, instead of being represented by the pair $(\boldsymbol{r}, \boldsymbol{p})$, elements of $\operatorname{Spin}(3) \ltimes \mathbb{R}^{3}$ are usually represented in a compact way by unit dual quaternions, which provides convenient ways of exploiting algebraic properties.

The set of dual quaternions is defined as

$$
\mathcal{H} \triangleq\left\{\boldsymbol{g}+\varepsilon \boldsymbol{h}: \boldsymbol{g}, \boldsymbol{h} \in \mathbb{H}, \varepsilon^{2}=0, \varepsilon \neq 0\right\},
$$


where $\varepsilon$ is the nilpotent Clifford unit, also known as dual unit (Selig, 2005). The dual quaternion conjugate of $\underline{\boldsymbol{h}}=\boldsymbol{g}+\varepsilon \boldsymbol{h}$ is $\underline{\boldsymbol{h}}^{*} \triangleq \boldsymbol{g}^{*}+\varepsilon \boldsymbol{h}^{*}$ and the dual quaternion norm is defined as $\|\underline{\boldsymbol{h}}\| \triangleq \sqrt{\underline{\boldsymbol{h}}} \underline{\boldsymbol{h}}^{*}$. Usually $\boldsymbol{g}$ and $\boldsymbol{h}$ are called primary part and dual part of $\underline{\boldsymbol{h}}$, respectively, and we define the operators

$$
\begin{aligned}
& \mathcal{P}(\underline{\boldsymbol{h}}) \triangleq \boldsymbol{g}, \\
& \mathcal{D}(\underline{\boldsymbol{h}}) \triangleq \boldsymbol{h}
\end{aligned}
$$

to retrieve those components from a dual quaternion.

Dual quaternions with unit norm are called unit dual quaternions and belong to the set

$$
\underline{\boldsymbol{S}} \triangleq\{\underline{\boldsymbol{h}} \in \mathcal{H}:\|\underline{\boldsymbol{h}}\|=1\} .
$$

Elements of $\underline{\boldsymbol{S}}$ equipped with the multiplication operation represent elements of $\operatorname{Spin}(3) \ltimes \mathbb{R}^{3}$, the group of rigid motions that double covers $S E$ (3). Let $r \in \operatorname{Spin}(3)$ and $\boldsymbol{p} \in \mathbb{H}_{p}$, the unit dual quaternion corresponding to the translation $\boldsymbol{p}$ followed by the rotation $\boldsymbol{r}$ is given by $\underline{\boldsymbol{x}}=\boldsymbol{r}+\varepsilon(1 / 2) \boldsymbol{p} \boldsymbol{r}$ and the inverse motion is given by the dual quaternion conjugate $\underline{x}^{*}$, which corresponds to the group inverse such that $\underline{\boldsymbol{x}}^{*} \underline{\boldsymbol{x}}=\underline{\boldsymbol{x}}^{*}=1$ (Selig, 2005). (Consequently, 1 is the group identity of $\operatorname{Spin}(3) \ltimes \mathbb{R}^{3}$.) Remarkably, the composition of rigid transformations is given by a sequence of dual quaternion multiplications. More specifically,

$$
\underline{\boldsymbol{x}}_{3} \triangleq\left[\boldsymbol{r}_{1}+\varepsilon \frac{1}{2} \boldsymbol{p}_{1} \boldsymbol{r}_{1}\right]\left[\boldsymbol{r}_{2}+\varepsilon \frac{1}{2} \boldsymbol{p}_{2} \boldsymbol{r}_{2}\right]=\boldsymbol{r}_{1} \boldsymbol{r}_{2}+\varepsilon \frac{1}{2}\left(\boldsymbol{r}_{1} \boldsymbol{p}_{2} \boldsymbol{r}_{2}+\boldsymbol{p}_{1} \boldsymbol{r}_{1} \boldsymbol{r}_{2}\right) .
$$

In order to see the equivalence between (4) and (2) first we start with the rotation, which is given by the primary part of the unit dual quaternion, $\mathcal{P}\left(\underline{\boldsymbol{x}}_{3}\right)=\boldsymbol{r}_{1} \boldsymbol{r}_{2}$ (here we see that the $\mathcal{P}(\cdot)$ operator, when applied to a unit dual quaternion, return elements of the subgroup of pure rotations). This is the same rotation of (2). To see that the translation of both equations is the same, we define the operator $\underline{\mathcal{T}}(\underline{\boldsymbol{x}})$ for $\underline{x} \in \operatorname{Spin}(3) \ltimes \mathbb{R}^{3}$ :

$$
\underline{\mathcal{I}}(\underline{x}) \triangleq \underline{x} \mathcal{P}(\underline{x})^{*}
$$

The $\underline{\mathcal{I}}$ operator, when applied to the unit dual quaternion $\underline{\boldsymbol{x}}$, returns its translation in dual quaternion form. Thus, this operator returns elements of the subgroup of pure translations. For example, given $\underline{\boldsymbol{x}}=\boldsymbol{r}+\varepsilon(1 / 2) \boldsymbol{p r}$,

$$
\begin{aligned}
\underline{\mathcal{T}}(\underline{\boldsymbol{x}}) & =\underline{\boldsymbol{x}} \mathcal{P}(\underline{\boldsymbol{x}})^{*}=\left(\boldsymbol{r}+\varepsilon \frac{1}{2} \boldsymbol{p r}\right) \boldsymbol{r}^{*} \\
& =1+\varepsilon \frac{1}{2} \boldsymbol{p} .
\end{aligned}
$$

This way, applying the $\underline{\mathcal{I}}$ operator to (4) we obtain

$$
\begin{aligned}
\underline{\mathcal{T}}\left(\underline{\boldsymbol{x}}_{3}\right) & =\left[\boldsymbol{r}_{1} \boldsymbol{r}_{2}+\varepsilon \frac{1}{2}\left(\boldsymbol{r}_{1} \boldsymbol{p}_{2} \boldsymbol{r}_{2}+\boldsymbol{p}_{1} \boldsymbol{r}_{1} \boldsymbol{r}_{2}\right)\right]\left(\boldsymbol{r}_{1} \boldsymbol{r}_{2}\right)^{*} \\
& =1+\varepsilon \frac{1}{2}\left(\operatorname{Ad}\left(\boldsymbol{r}_{1}\right) \boldsymbol{p}_{2}+\boldsymbol{p}_{1}\right),
\end{aligned}
$$

which corresponds to the translation of (2). 


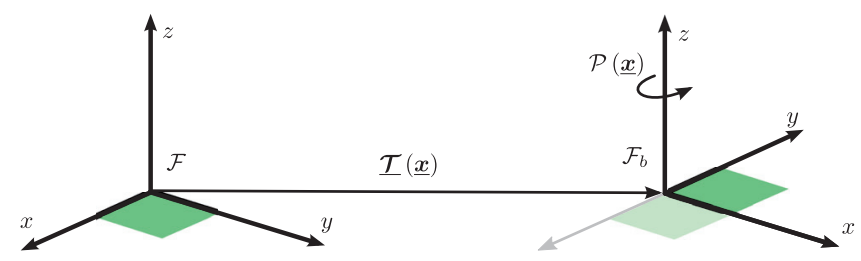

FIG. 1. The unit dual quaternion $\underline{\boldsymbol{x}}=\underline{\mathcal{T}}(\underline{\boldsymbol{x}}) \mathcal{P}(\underline{\boldsymbol{x}})$ represents the rigid transformation from the inertial frame $\mathcal{F}$ to $\mathcal{F}_{b}$.

From (5) we see that $\underline{x}=\underline{\mathcal{T}}(\underline{\boldsymbol{x}}) \mathcal{P}(\underline{\boldsymbol{x}})$, which means that if $\underline{\boldsymbol{x}}$ represents the rigid transformation from a inertial frame $\mathcal{F}$ to frame $\mathcal{F}_{b}$ then $\underline{x}$ can be interpreted as a sequence of two transformations: first a transformation $\underline{\mathcal{T}}(\underline{\boldsymbol{x}})$ that translates the origin of $\mathcal{F}$ to the origin of frame $\mathcal{F}_{b}$ and then a transformation $\mathcal{P}(\underline{\boldsymbol{x}})$ that rotates the translated frame in order to align it with $\mathcal{F}_{b}$, as shown in Fig. 1. Any subsequent rigid transformation, when performed by a right multiplication, uses $\mathcal{F}_{b}$ as the reference frame for the transformation. However, in case a subsequent rigid transformation is performed by a left multiplication, the inertial frame $\mathcal{F}$ is used as reference frame for the transformation.

\subsection{Motivation}

Because of the construction of $\operatorname{Spin}(3) \ltimes \mathbb{R}^{3}$, the final translation after a sequence of rigid motions always depends on the intermediate rotations (note that this is true also for $S E(3)$ ). This dependence, which is intrinsic to Spin $(3) \ltimes \mathbb{R}^{3}$, can be called cross-motion variance, and has important consequences when describing rigid motions. For instance, some specific motions cannot be directly specified, without intermediate transformations, with respect to arbitrary frames when only $\operatorname{Spin}(3) \ltimes \mathbb{R}^{3}$ operations are used.

In order to see this fact, consider a manipulator robot and let $\underline{x}_{0} \in \operatorname{Spin}(3) \ltimes \mathbb{R}^{3}$ be the initial pose of the frame $\mathcal{F}_{E}$ attached to the end effector with respect to the base frame $\mathcal{F}_{b}$ (Fig. 2). A rigid motion $\underline{\boldsymbol{m}}_{E} \in \operatorname{Spin}(3) \ltimes \mathbb{R}^{3}$ can be specified with respect to the end-effector frame $\mathcal{F}_{E}$ by performing a right multiplication on $\underline{\boldsymbol{x}}_{0}$, that is $\underline{\boldsymbol{x}}_{1}=\underline{\boldsymbol{x}}_{0} \underline{\boldsymbol{m}}_{E}$, where $\underline{\boldsymbol{x}}_{1}$ is the pose of the end effector after the motion $\underline{\boldsymbol{m}}_{E}$. Alternatively, an equivalent motion $\underline{\boldsymbol{m}}_{B}$ can be specified with respect to the base frame $\mathcal{F}_{B}$ to generate the same final end-effector pose; that is,

$$
\underline{\boldsymbol{x}}_{1}=\underline{\boldsymbol{m}}_{B} \underline{\boldsymbol{x}}_{0}
$$

Since $\underline{\boldsymbol{x}}_{0} \underline{\boldsymbol{m}}_{E}=\underline{\boldsymbol{x}}_{1}=\underline{\boldsymbol{m}}_{B} \underline{\boldsymbol{x}}_{0}$, then

$$
\underline{\boldsymbol{m}}_{B}=\underline{\boldsymbol{x}}_{0} \underline{\boldsymbol{m}}_{E} \underline{x}_{0}^{*} .
$$

Let us consider a motion $\underline{\boldsymbol{m}}_{E} \triangleq \boldsymbol{r}_{E}$ that consists of just a rotation of the end effector using $\mathcal{F}_{E}$ as the reference frame. The final end-effector pose is

$$
\underline{\boldsymbol{x}}_{1}=\underline{\boldsymbol{x}}_{0} \boldsymbol{r}_{E}
$$

and $\underline{\mathcal{I}}\left(\underline{\boldsymbol{x}}_{1}\right)=\underline{\mathcal{I}}\left(\underline{\boldsymbol{x}}_{0}\right)$, which implies that the end-effector position does not change. However, if the motion is to be described with respect to the base frame but resulting in the same final end-effector pose, the solution given by (7) is 


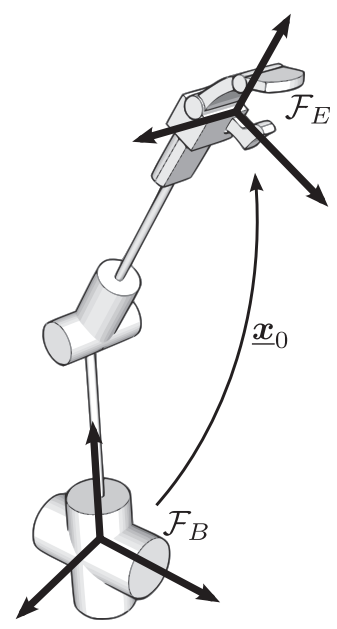

FIG. 2. Common frames in manipulator robots: $\mathcal{F}_{B}$ is the base frame and $\mathcal{F}_{E}$ is the end-effector frame. The unit dual quaternion $\underline{x}_{0}$ corresponds to the initial pose of the robot end effector with respect to the base frame.

$$
\underline{\boldsymbol{x}}_{1}=\left(\underline{\boldsymbol{x}}_{0} \boldsymbol{r}_{E} \underline{x}_{0}^{*}\right) \underline{\boldsymbol{x}}_{0},
$$

which yields exactly (8); that is, using the group operation of $\operatorname{Spin}(3) \ltimes \mathbb{R}^{3}$, a movement of rotation without translation must be essentially specified with respect to the end-effector frame. Otherwise, if the movement is specified directly with respect to the base frame, one must also take into account the coupling between rotations and translations and then specify a suitable translation in order to maintain the end-effector position constant, which can be quite counterintuitive.

To better clarify this point, consider that the desired motion is a pure rotation $\underline{\boldsymbol{m}}_{B}=\boldsymbol{r}_{B}$ of the end effector but defined with respect to $\mathcal{F}_{B}$. The result of $\underline{\boldsymbol{m}}_{B} \boldsymbol{x}_{0}$ is not the desired one, since $\underline{\mathcal{T}}\left(\underline{\boldsymbol{m}}_{B} \boldsymbol{x}_{0}\right)=$ $1+\varepsilon(1 / 2) \operatorname{Ad}\left(\boldsymbol{r}_{B}\right) \boldsymbol{p}_{0}$, which is different from $\underline{\mathcal{T}}\left(\underline{\boldsymbol{x}}_{0}\right)=1+\varepsilon(1 / 2) \boldsymbol{p}_{0}$ (although at least the final orientation is the desired one). Actually, the desired value for $\underline{\boldsymbol{m}}_{B}$ such that $\underline{\mathcal{T}}\left(\underline{\boldsymbol{m}}_{B} \underline{\boldsymbol{x}}_{0}\right)=\underline{\mathcal{T}}\left(\underline{\boldsymbol{x}}_{0}\right)$ is $\underline{\boldsymbol{m}}_{B}=\boldsymbol{r}_{B}+\varepsilon(1 / 2)\left[\boldsymbol{p}_{0}-\operatorname{Ad}\left(\boldsymbol{r}_{B}\right) \boldsymbol{p}_{0}\right] \boldsymbol{r}_{B}$. Not only this transformation is counterintuitive but it also has a fundamental problem since it depends on the initial position of the end effector and this position may be uncertain. This way, because of the uncertainty of $\boldsymbol{p}_{0}$ the desired value of $\underline{\boldsymbol{m}}_{B}$ is also uncertain, which does not happen when the task is defined at the end-effector frame.

It is important to highlight that those issues arise not only in the context of manipulator robots but in any type of kinematic chain. It suffices to have different coordinate systems in which some of them are inertial and others are moving and motions must be specified in different frames, which is very typical in cooperative robot systems. In this context, it would be much more convenient if there existed a more intuitive operation, for example $\otimes$, acting on $\operatorname{Spin}(3) \ltimes \mathbb{R}^{3}$ operands such that rigid motions could be defined more intuitively, independently of the choice of coordinate system; for instance, it would be convenient to describe the motion of rotation without translation, defined at the base frame, as $\boldsymbol{x}_{1}=\boldsymbol{r}_{B} \otimes \underline{\boldsymbol{x}}_{0}=\left[1+\varepsilon(1 / 2) \boldsymbol{p}_{0}\right] \boldsymbol{r}_{B} \boldsymbol{r}_{0}$. More generally, given $\underline{\boldsymbol{x}}_{1}=\boldsymbol{r}_{1}+\varepsilon(1 / 2) \boldsymbol{p}_{1} \boldsymbol{r}_{1}$ and $\underline{\boldsymbol{x}}_{2}=\boldsymbol{r}_{2}+\varepsilon(1 / 2) \boldsymbol{p}_{2} \boldsymbol{r}_{2}$, we seek an operation $\otimes$ such that

$$
\underline{\boldsymbol{x}}_{1} \otimes \underline{\boldsymbol{x}}_{2}=\boldsymbol{r}_{1} \boldsymbol{r}_{2}+\varepsilon \frac{1}{2}\left(\boldsymbol{p}_{1}+\boldsymbol{p}_{2}\right) \boldsymbol{r}_{1} \boldsymbol{r}_{2}
$$


In terms of groups, this is achievable simply by taking the direct product of $\operatorname{Spin}(3)$ and $\mathbb{R}^{3}$, that is $\operatorname{Spin}(3) \times \mathbb{R}^{3}$. However, more than just a convenient notation such operation should provide useful features to describe motions that cannot be easily described using $\operatorname{Spin}(3) \ltimes \mathbb{R}^{3}$ operations. Moreover, this new operation should be complementary to the standard one in order to enrich the algebra of rigid motions.

In this article, we propose a new group, the Cross Motion Invariant group $C M I(3)$, which has the following characteristics: (i) rotation operations do not affect translations and vice-versa (cross-motion invariance), (ii) motions are invariant with respect to the position of the reference frame, (iii) translations under $C M I(3)$ operations commute with arbitrary rigid motions and (iv) the direction of trajectories is preserved under $C M I(3)$ operations. Furthermore $C M I(3)$ operations can be naturally mixed with $\operatorname{Spin}(3) \ltimes \mathbb{R}^{3}$ operations, leading to a richer algebra and an abstraction level that can be quite useful when describing rigid motions. Also, we derive the kinematic equation of a sequence of rigid motions under the $C M I(3)$ group operation.

This article is organized as follows: the next section presents the group $C M I(3)$ and its underlying operation, the decompositional multiplication, along with the corresponding geometrical interpretation. Section 3 presents the kinematic equation of a sequence of rigid motions under $C M I(3)$ operations, whereas Section 4 presents two numerical examples to illustrate the behaviour of rigid motions under $C M I(3)$ operations. Lastly, Section 5 concludes the article.

\section{The group $C M I(3)$ and decompositional multiplications}

Before formally introducing the group $C M I(3)$, let us first recall that a group is a manifold $G$ equipped with an identity element $e$ and two operations

$$
\text { mult }: G \times G \rightarrow G, \quad \text { inv }: G \rightarrow G,
$$

such that mult, also known as the group operation, must be associative and obey the closure property and inv must be a bijection (Selig, 2005). So, in order to define the group CMI(3) first we must establish the underlying manifold of unit dual quaternions and then we must define the group operation, the group identity and the group inverse.

The underlying manifold of unit dual quaternions is $\mathbb{S}^{3} \times \mathbb{R}^{3}$, where $\mathbb{S}^{3}$ is the unit 3-sphere in fourdimensional space and thus the set $\mathcal{S}$ of unit dual quaternions 'is a six-dimensional algebraic submanifold of $\mathbb{R}^{8}$, (McCarthy, 1990). Since $C M I(3)$ is a group acting on unit dual quaternions, thus $G \triangleq \mathbb{S}^{3} \times \mathbb{R}^{3}$. The group operation of $C M I(3)$ is denoted by $\otimes$ to distinguish it from the standard multiplication of $\operatorname{Spin}(3) \ltimes \mathbb{R}^{3}$ and is defined in the following.

Definition 1 Given $\underline{\boldsymbol{x}}_{a}, \underline{\boldsymbol{x}}_{t} \in \underline{\mathcal{S}}$, the decompositional multiplication, represented by $\otimes$, is defined as

$$
\underline{\boldsymbol{x}}_{a} \otimes \underline{\boldsymbol{x}}_{t} \triangleq \underline{\mathcal{T}}\left(\underline{\boldsymbol{x}}_{a}\right) \underline{\mathcal{T}}\left(\underline{\boldsymbol{x}}_{t}\right) \mathcal{P}\left(\underline{\boldsymbol{x}}_{a}\right) \mathcal{P}\left(\underline{\boldsymbol{x}}_{t}\right) .
$$

Lemma 1 (Closure) The set $\underline{\mathcal{S}}$ is closed under the decompositional multiplication.

Proof. Let $\underline{\boldsymbol{x}}_{a}, \underline{\boldsymbol{x}}_{t} \in \underline{\mathcal{S}}$ and consider $\underline{\boldsymbol{x}}_{f} \triangleq \underline{\boldsymbol{x}}_{a} \otimes \underline{\boldsymbol{x}}_{t}=\underline{\mathcal{I}}\left(\underline{\boldsymbol{x}}_{a}\right) \underline{\mathcal{T}}\left(\underline{\boldsymbol{x}}_{t}\right) \mathcal{P}\left(\underline{\boldsymbol{x}}_{a}\right) \mathcal{P}\left(\underline{\boldsymbol{x}}_{t}\right)$, then

$$
\underline{\boldsymbol{x}}_{f} \underline{\boldsymbol{x}}_{f}^{*}=\underline{\boldsymbol{T}}\left(\underline{\boldsymbol{x}}_{a}\right) \underline{\mathcal{T}}\left(\underline{\boldsymbol{x}}_{t}\right) \mathcal{P}\left(\underline{\boldsymbol{x}}_{a}\right) \mathcal{P}\left(\underline{\boldsymbol{x}}_{t}\right) \mathcal{P}\left(\underline{\boldsymbol{x}}_{t}\right)^{*} \mathcal{P}\left(\underline{\boldsymbol{x}}_{a}\right)^{*} \underline{\mathcal{T}}\left(\underline{\boldsymbol{x}}_{t}\right)^{*} \underline{\mathcal{T}}\left(\underline{\boldsymbol{x}}_{a}\right)^{*}
$$


If $\underline{\boldsymbol{x}} \in \underline{\mathcal{S}}$ then $\mathcal{P}(\underline{\boldsymbol{x}}) \mathcal{P}(\underline{\boldsymbol{x}})^{*}=1$ and $\underline{\mathcal{T}}(\underline{\boldsymbol{x}}) \underline{\mathcal{T}}(\underline{\boldsymbol{x}})^{*}=1$, hence $\underline{\boldsymbol{x}}_{f} \underline{\boldsymbol{x}}_{f}^{*}=1$, which implies $\left\|\underline{\boldsymbol{x}}_{f}\right\|=1$. Thus $\underline{\boldsymbol{x}}_{f} \in \underline{\mathcal{S}}$.

Lemma 2 Let $\underline{\boldsymbol{x}}_{1}, \underline{\boldsymbol{x}}_{2} \in \underline{\mathcal{S}}$, then $\underline{\mathcal{T}}\left(\underline{\boldsymbol{x}}_{1} \otimes \underline{\boldsymbol{x}}_{2}\right)=\underline{\mathcal{T}}\left(\underline{\boldsymbol{x}}_{1}\right) \underline{\boldsymbol{T}}\left(\underline{\boldsymbol{x}}_{2}\right)$ and $\mathcal{P}\left(\underline{\boldsymbol{x}}_{1} \otimes \underline{\boldsymbol{x}}_{2}\right)=\mathcal{P}\left(\underline{\boldsymbol{x}}_{1}\right) \mathcal{P}\left(\underline{\boldsymbol{x}}_{2}\right)$.

Proof. Considering that a unit dual quaternion $\underline{\boldsymbol{x}}_{i}$ can always be written as $\underline{\boldsymbol{x}}_{i}=\boldsymbol{r}_{i}+\varepsilon(1 / 2) \boldsymbol{p}_{i} \boldsymbol{r}_{i}$, then

$$
\begin{aligned}
\underline{\boldsymbol{x}}_{1} \otimes \underline{\boldsymbol{x}}_{2} & =\left(1+\varepsilon \frac{1}{2} \boldsymbol{p}_{1}\right)\left(1+\varepsilon \frac{1}{2} \boldsymbol{p}_{2}\right) \boldsymbol{r}_{1} \boldsymbol{r}_{2} \\
& =\boldsymbol{r}_{1} \boldsymbol{r}_{2}+\varepsilon \frac{1}{2}\left(\boldsymbol{p}_{1}+\boldsymbol{p}_{2}\right) \boldsymbol{r}_{1} \boldsymbol{r}_{2} .
\end{aligned}
$$

Thus,

$$
\underline{\mathcal{T}}\left(\underline{\boldsymbol{x}}_{1} \otimes \underline{\boldsymbol{x}}_{2}\right)=1+\varepsilon \frac{1}{2}\left(\boldsymbol{p}_{1}+\boldsymbol{p}_{2}\right)=\underline{\mathcal{T}}\left(\underline{\boldsymbol{x}}_{1}\right) \underline{\mathcal{T}}\left(\underline{\boldsymbol{x}}_{2}\right)
$$

and

$$
\mathcal{P}\left(\underline{\boldsymbol{x}}_{1} \otimes \underline{\boldsymbol{x}}_{2}\right)=\boldsymbol{r}_{1} \boldsymbol{r}_{2}=\mathcal{P}\left(\underline{\boldsymbol{x}}_{1}\right) \mathcal{P}\left(\underline{\boldsymbol{x}}_{2}\right)
$$

Lemma 3 (Associativity) The decompositional multiplication is associative.

Proof. Considering $\underline{\boldsymbol{x}}_{1}, \underline{\boldsymbol{x}}_{2}, \underline{\boldsymbol{x}}_{3} \in \underline{\mathcal{S}}$ and using Lemma 2,

$$
\begin{aligned}
\left(\underline{\boldsymbol{x}}_{1} \otimes \underline{\boldsymbol{x}}_{2}\right) \otimes \underline{\boldsymbol{x}}_{3} & =\underline{\mathcal{T}}\left(\underline{\boldsymbol{x}}_{1} \otimes \underline{\boldsymbol{x}}_{2}\right) \underline{\mathcal{T}}\left(\underline{\boldsymbol{x}}_{3}\right) \mathcal{P}\left(\underline{\boldsymbol{x}}_{1} \otimes \underline{\boldsymbol{x}}_{2}\right) \mathcal{P}\left(\underline{\boldsymbol{x}}_{3}\right) \\
& =\underline{\mathcal{T}}\left(\underline{\boldsymbol{x}}_{1}\right) \underline{\mathcal{T}}\left(\underline{\boldsymbol{x}}_{2}\right) \underline{\mathcal{T}}\left(\underline{\boldsymbol{x}}_{3}\right) \mathcal{P}\left(\underline{\boldsymbol{x}}_{1}\right) \mathcal{P}\left(\underline{\boldsymbol{x}}_{2}\right) \mathcal{P}\left(\underline{\boldsymbol{x}}_{3}\right) \\
& =\underline{\mathcal{T}}\left(\underline{\boldsymbol{x}}_{1}\right) \underline{\mathcal{T}}\left(\underline{\boldsymbol{x}}_{2} \otimes \underline{\boldsymbol{x}}_{3}\right) \mathcal{P}\left(\underline{\boldsymbol{x}}_{1}\right) \mathcal{P}\left(\underline{\boldsymbol{x}}_{2} \otimes \underline{\boldsymbol{x}}_{3}\right) \\
& =\underline{\boldsymbol{x}}_{1} \otimes\left(\underline{\boldsymbol{x}}_{2} \otimes \underline{\boldsymbol{x}}_{3}\right) .
\end{aligned}
$$

LEMMA 4 (GROUP IDENTITY) The identity element under the decompositional multiplication is the real number 1 .

Proof. First, it is clear that $1 \in \mathcal{S}$, since $\mathbb{R} \subset \mathbb{C} \subset \mathbb{H} \subset \mathcal{H}$ and $\|1\|=1$. Also, $\mathcal{P}(1)=1$ hence $\underline{\mathcal{I}}(1)=1$. Thus, considering any $\underline{\boldsymbol{x}} \in \underline{\mathcal{S}}$ we have that $\underline{\boldsymbol{x}} \otimes 1=1 \otimes \underline{\boldsymbol{x}}=\underline{\mathcal{I}}(\underline{\boldsymbol{x}}) \mathcal{P}(\underline{\boldsymbol{x}})=\underline{\boldsymbol{x}}$.

LEMMA 5 (GROUP INVERSE) Given $\underline{\boldsymbol{x}} \in \underline{\mathcal{S}}$, the inverse operation under decompositional multiplication is a bijective mapping given by

$$
\underline{\boldsymbol{x}}^{\dagger}=\underline{\mathcal{T}}(\underline{\boldsymbol{x}})^{*} \mathcal{P}(\underline{\boldsymbol{x}})^{*}
$$

Proof. Since $\underline{\mathcal{T}}\left(\underline{\boldsymbol{x}}^{\dagger}\right)=\underline{\mathcal{T}}(\underline{\boldsymbol{x}})^{*}$ and $\mathcal{P}\left(\underline{\boldsymbol{x}}^{\dagger}\right)=\mathcal{P}(\underline{\boldsymbol{x}})^{*}$, then we verify by direct calculation that

$$
\underline{\boldsymbol{x}}^{\dagger} \otimes \underline{\boldsymbol{x}}=\underline{\boldsymbol{x}} \otimes \underline{\boldsymbol{x}}^{\dagger}=1 .
$$


In addition, we see that

$$
\left(\underline{x}^{\dagger}\right)^{\dagger}=\underline{\mathcal{I}}\left(\underline{x}^{\dagger}\right)^{*} \mathcal{P}\left(\underline{x}^{\dagger}\right)^{*}=\underline{\mathcal{T}}(\underline{x}) \mathcal{P}(\underline{x})=\underline{x} .
$$

In order to show that the inverse operation is injective, consider $\underline{x}_{1}, \underline{x}_{2} \in \underline{\mathcal{S}}$ such that $\underline{x}_{1} \neq \underline{\boldsymbol{x}}_{2}$ but $\underline{x}_{1}^{\dagger}=\underline{\boldsymbol{x}}_{2}^{\dagger}$. Inverting both sides of the equality gives $\left(\underline{\boldsymbol{x}}_{1}^{\dagger}\right)^{\dagger}=\left(\underline{\boldsymbol{x}}_{2}^{\dagger}\right)^{\dagger}$, which implies from (12) that $\underline{\boldsymbol{x}}_{1}=\underline{\boldsymbol{x}}_{2}$, which is a contradiction. Hence the inverse operation under decompositional multiplication is injective.

In order to show that the inverse operation is surjective, let us assume that there exists a unit dual quaternion $\underline{\boldsymbol{a}}$ in the codomain of the inverse operation but not in its image. Because (11) is defined for all unit dual quaternions, there is a unit dual quaternion $\underline{\boldsymbol{a}}$ in the domain such that $\underline{a}^{\dagger}=\underline{\boldsymbol{b}}$. Since the domain also contains $\underline{\boldsymbol{b}}$, then $\underline{\boldsymbol{b}}^{\dagger}=\left(\underline{\boldsymbol{a}}^{\dagger}\right)^{\dagger}=\underline{\boldsymbol{a}}$; that is, $\underline{\boldsymbol{a}}$ must be in the image of the inverse operation, contradicting the initial claim that $\underline{\boldsymbol{a}}$ is in the codomain of the inverse operation, but not in its image, thus implying surjectivity.

THEOREM 1 The set $\underline{\mathcal{S}}$ of unit dual quaternions under the decompositional multiplication form a group.

Proof. First, $\mathcal{S}$ is a manifold $\left(\mathbb{S}^{3} \times \mathbb{R}^{3}\right.$, see McCarthy, 1990) and the decompositional multiplication is closed and associative (Lemmas 1 and 3). In addition, there is an identity element under the decompositional multiplication (Lemma 4) and the inverse operation is a bijection (Lemma 5), consequently unit dual quaternions under decompositional multiplication form a group, which is named $C M I(3)$.

\subsection{Geometrical interpretation of $\mathrm{CMI}(3)$ operations}

Note that both operators $\underline{\mathcal{I}}(\cdot)$ and $\mathcal{P}(\cdot)$ return unit dual quaternions, the former corresponding to a pure translation and the latter corresponding to a pure rotation. This way, the decompositional multiplication corresponds to a rigid motion that behaves differently from the rigid motion performed by the standard multiplication of $\operatorname{Spin}(3) \ltimes \mathbb{R}^{3}$, which is given by

$$
\underline{\boldsymbol{x}}_{a} \underline{\boldsymbol{x}}_{t}=\underline{\mathcal{T}}\left(\underline{\boldsymbol{x}}_{a}\right) \mathcal{P}\left(\underline{\boldsymbol{x}}_{a}\right) \underline{\mathcal{T}}\left(\underline{\boldsymbol{x}}_{t}\right) \mathcal{P}\left(\underline{\boldsymbol{x}}_{t}\right) .
$$

In order to illustrate this difference, let $\underline{\boldsymbol{x}}_{t}$ and $\underline{\boldsymbol{x}}_{a}$ be unit dual quaternions such that $\underline{\boldsymbol{x}}_{t}$ represents a target frame $\mathcal{F}_{t}$ (which could be attached to any relevant rigid body, for example an object to be manipulated or the robot end effector) with respect to the inertial frame $\mathcal{F}$ and $\underline{\boldsymbol{x}}_{a}$ represents a transformation that will be applied on $\underline{\boldsymbol{x}}_{t}$. In addition, let $\underline{\mathcal{T}}\left(\underline{\boldsymbol{x}}_{t}\right)=1+\varepsilon(\hat{\jmath} d) / 2$ and $\mathcal{P}\left(\underline{\boldsymbol{x}}_{t}\right)=\cos (\pi / 4)+\hat{k} \sin (\pi / 4)$; that is, considering the reference frame $\mathcal{F}, \underline{x}_{t}$ is the pose of the target frame obtained after a translation $d$ along the $y$ axis followed by a rotation of $\pi / 2$ around the $z$ axis. Also, consider $\underline{\mathcal{T}}\left(\underline{x}_{a}\right)=1+\varepsilon(\hat{\jmath} d) / 2$ and $\mathcal{P}\left(\underline{x}_{a}\right)=\cos (\pi / 4)+\hat{\imath} \sin (\pi / 4)$; that is, the transformation $\underline{x}_{a}$ corresponds to anslation $d$ along the $y$ axis and a rotation of $\pi / 2$ around the $x$ axis.

The reference frame for the transformation $\underline{x}_{a}$ defines if it corresponds to a left multiplication or a right multiplication: if the target frame is used as reference, right multiplication is used (i.e. $\underline{\boldsymbol{x}}_{t} \underline{\boldsymbol{x}}_{a}$ in case of the standard multiplication and $\underline{\boldsymbol{x}}_{t} \otimes \underline{\boldsymbol{x}}_{a}$ in case of the decompositional multiplication); conversely, if the inertial frame is the reference for the transformation, left multiplication is used (i.e. $\underline{\boldsymbol{x}}_{a} \underline{\boldsymbol{x}}_{t}$ in case of the standard multiplication and $\underline{\boldsymbol{x}}_{a} \otimes \underline{\boldsymbol{x}}_{t}$ in case of the decompositional multiplication).

Assuming that the desired transformation is given with respect to the inertial frame $\mathcal{F}$, the resulting pose under the standard $\operatorname{Spin}(3) \ltimes \mathbb{R}^{3}$ multiplication, given by (13), can be interpreted as follows. Given 
(a)

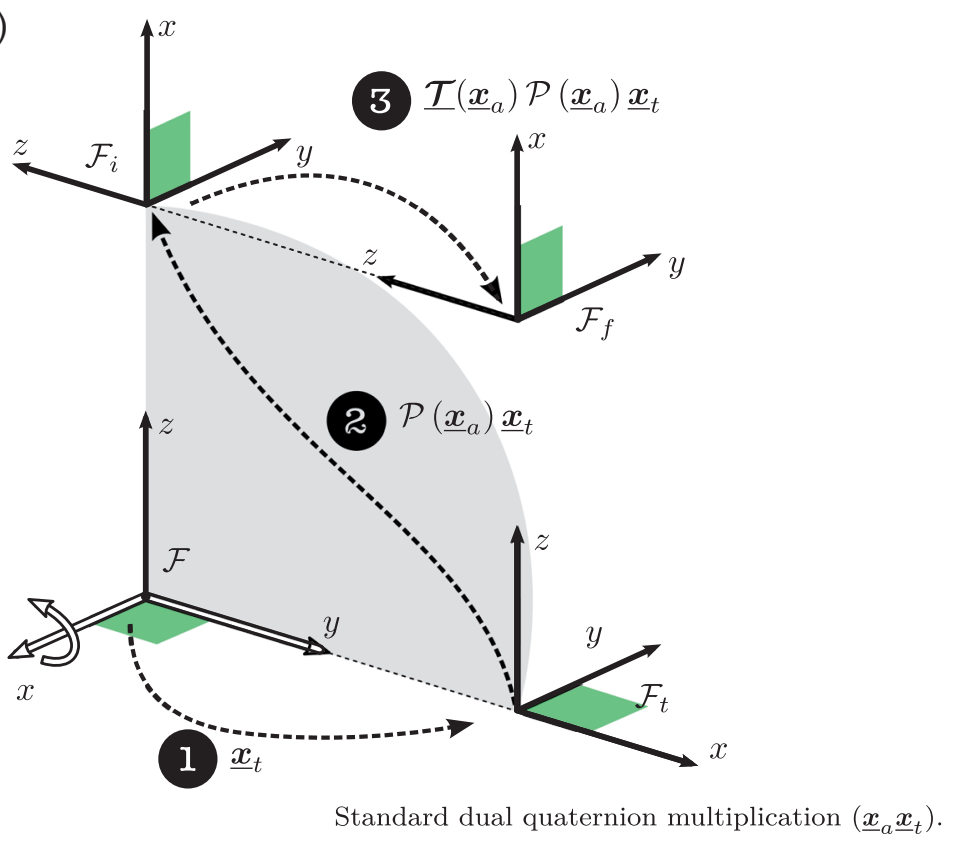

(b)

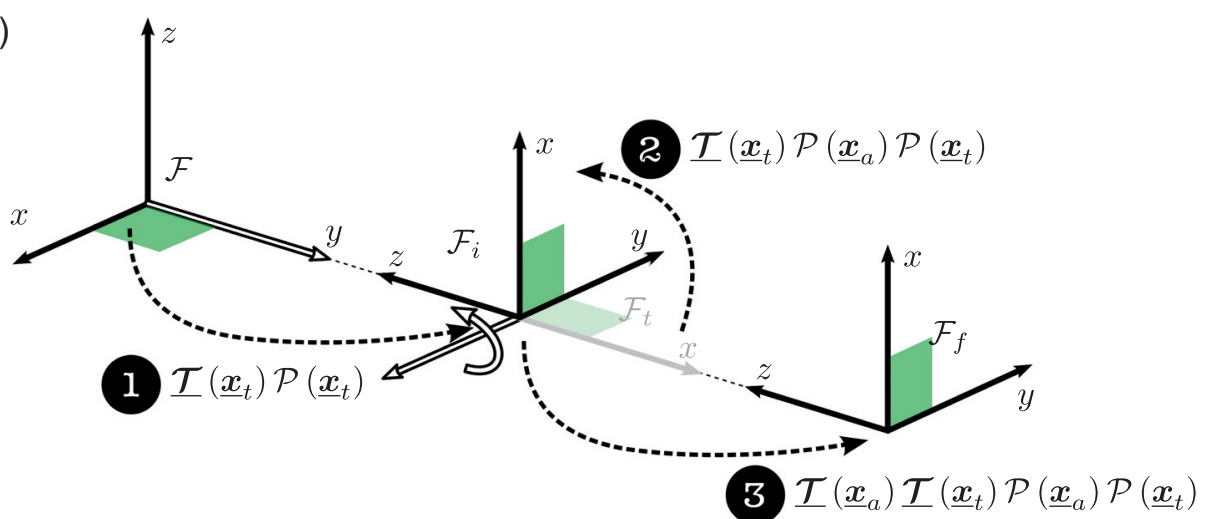

Decompositional dual quaternion multiplication $\left(\underline{\boldsymbol{x}}_{a} \otimes \underline{\boldsymbol{x}}_{t}\right)$

FIG. 3. $\mathcal{F}$ is the inertial frame, $\mathcal{F}_{i}$ is an intermediate frame and $\mathcal{F}_{t}$ is the target frame. The transformation $\underline{\mathcal{T}}\left(\underline{\boldsymbol{x}}_{a}\right)$ corresponds to a translation $d$ along the $y$ axis and $\mathcal{P}\left(\underline{x}_{a}\right)$ corresponds to a rotation of $\pi / 2$ around the $x$ axis. The contour arrows are the ones used to perform the translation and rotation. Each numbered circle corresponds to one intermediate step of the complete transformation.

$\underline{\boldsymbol{x}}_{t}$ (see step 1 in Fig. 3a), first we rotate it by applying $\mathcal{P}\left(\underline{\boldsymbol{x}}_{a}\right) \underline{\mathcal{T}}\left(\underline{\boldsymbol{x}}_{t}\right) \mathcal{P}\left(\underline{\boldsymbol{x}}_{t}\right)$ resulting in the intermediate frame $\mathcal{F}_{i}$ (step 2 in Fig. 3a). Since this is a left multiplication, the $x$-axis of the inertial frame $\mathcal{F}$ is used for the transformation (see bent contour arrow in Fig. 3a). Then we translate $\mathcal{F}_{i}$ by applying the last transformation, $\underline{\mathcal{T}}\left(\underline{\boldsymbol{x}}_{a}\right) \mathcal{P}\left(\underline{\boldsymbol{x}}_{a}\right) \underline{\mathcal{T}}\left(\underline{\boldsymbol{x}}_{t}\right) \mathcal{P}\left(\underline{\boldsymbol{x}}_{t}\right)$, which results in the final frame $\mathcal{F}_{f}$ (step 3 in Fig. 3a). Again, since this is a left multiplication, the $y$ axis of the inertial frame $\mathcal{F}$ is used for the transformation. 
On the other hand, the resulting pose under the $C M I(3)$ multiplication given by (10) can be interpreted as follows. Given $\underline{\boldsymbol{x}}_{t}=\underline{\mathcal{I}}\left(\underline{\boldsymbol{x}}_{t}\right) \mathcal{P}\left(\underline{\boldsymbol{x}}_{t}\right)$ (see step 1 in Fig. 3b), first we rotate it by applying $\underline{\mathcal{I}}\left(\underline{\boldsymbol{x}}_{t}\right) \mathcal{P}\left(\underline{\boldsymbol{x}}_{a}\right) \mathcal{P}\left(\underline{\boldsymbol{x}}_{t}\right)$ resulting in the intermediate frame $\mathcal{F}_{i}$ (see step 2 in Fig. 3b). Differently from the standard multiplication, the transformation $\mathcal{P}\left(\underline{\boldsymbol{x}}_{a}\right)$ acts locally, as the reference for this transformation is a frame aligned with $\mathcal{F}$ but with the same origin of $\mathcal{F}_{t}$. (Note that $\underline{\mathcal{I}}\left(\underline{\boldsymbol{x}}_{t}\right)$ 'brings' the origin of the reference frame to the origin of $\mathcal{F}_{t}$ without changing the alignment, as $\underline{\mathcal{I}}(\cdot)$ corresponds to a pure translation.) The result is that the orientation of the resulting frame $\mathcal{F}_{i}$ is different from the orientation of $\mathcal{F}_{t}$ but the position is the same. Finally, $\mathcal{F}_{i}$ is translated by applying the last transformation $\underline{\mathcal{T}}\left(\underline{\boldsymbol{x}}_{a}\right) \underline{\mathcal{T}}\left(\underline{\boldsymbol{x}}_{t}\right) \mathcal{P}\left(\underline{\boldsymbol{x}}_{a}\right) \mathcal{P}\left(\underline{\boldsymbol{x}}_{t}\right)$ resulting in the final frame $\mathcal{F}_{f}$ (see step 3 in Fig. 3b). Again, since this is a left multiplication, the $y$ axis of the inertial frame $\mathcal{F}$ is used for the transformation.

REMARK 1 Note that in Fig 3 a the position of the final frame clearly depends on the rotation $\mathcal{P}\left(\underline{\boldsymbol{x}}_{a}\right)$. Even if the action of $\underline{\boldsymbol{x}}_{a}$ on $\underline{\boldsymbol{x}}_{t}$ were applied by the right side (i.e. $\underline{\boldsymbol{x}}_{f}=\underline{\boldsymbol{x}}_{t} \underline{\boldsymbol{x}}_{a}$ ), the final position of $\mathcal{F}_{f}$ would depend on the initial orientation of $\mathcal{F}_{t}$. The influence of rotation on translation can be called cross-motion variance. This is a property intrinsic to the group $\operatorname{Spin}(3) \ltimes \mathbb{R}^{3}$ (and also of $S E(3)$ ). On the other hand, decompositional multiplication is cross motion invariant, meaning that the translation will never be affected by the rotation part of the operation and vice versa, as shown in Fig. $3 \mathrm{~b}$. If the action were applied by the right (i.e. $\underline{\boldsymbol{x}}_{f}=\underline{\boldsymbol{x}}_{t} \otimes \underline{\boldsymbol{x}}_{a}$ ), the initial orientation of $\mathcal{F}_{t}$ would not affect the final position of $\mathcal{F}_{f}$. This is a property that cannot be achieved by using the standard group operation of $\operatorname{Spin}(3) \ltimes \mathbb{R}^{3}$ (and also of $S E(3)$ ).

REMARK 2 For any transformation under the $C M I(3)$ multiplication, there is a corresponding sequence of transformations given by standard $\operatorname{Spin}(3) \ltimes \mathbb{R}^{3}$ multiplications. For instance, consider a target frame $\mathcal{F}_{t}$ with the corresponding unit dual quaternion $\underline{\boldsymbol{x}}_{t}$ and a transformation $\boldsymbol{y} \in C M I(3)$, such that the final transformation is given by $\underline{\boldsymbol{x}}_{f}=\underline{\boldsymbol{y}} \otimes \underline{\boldsymbol{x}}_{t}$. Now, consider that we want to find a corresponding transformation $\underline{z} \in \operatorname{Spin}(3) \ltimes \mathbb{R}^{3}$ that results in the same final modified frame, that is, $\underline{\boldsymbol{x}}_{f}=\underline{z}_{-}$. Consequently,

$$
\begin{aligned}
\underline{\boldsymbol{z}}_{t} & =\underline{\boldsymbol{y}}^{\otimes} \underline{\boldsymbol{x}}_{t} \\
\Longrightarrow \underline{\boldsymbol{z}} & =\left(\underline{\boldsymbol{y}} \otimes \underline{\boldsymbol{x}}_{t}\right) \underline{\boldsymbol{x}}_{t}^{*} \\
& =\underline{\boldsymbol{T}}(\underline{\boldsymbol{y}}) \underline{\mathcal{T}}\left(\underline{\boldsymbol{x}}_{t}\right) \mathcal{P}\left(\underline{\boldsymbol{y}}^{\prime}\right) \mathcal{P}\left(\underline{\boldsymbol{x}}_{t}\right) \mathcal{P}\left(\underline{\boldsymbol{x}}_{t}\right)^{*} \underline{\mathcal{T}}\left(\underline{\boldsymbol{x}}_{t}\right)^{*} \\
& =\underline{\boldsymbol{T}}(\underline{\boldsymbol{y}}) \underline{\mathcal{T}}\left(\underline{\boldsymbol{x}}_{t}\right) \mathcal{P}(\underline{\boldsymbol{y}}) \underline{\mathcal{T}}\left(\underline{\boldsymbol{x}}_{t}\right)^{*} .
\end{aligned}
$$

This way, both operations can be used together because the result is always a unit dual quaternion. The choice of a particular operation depends mostly on the desired final transformation and which one provides a more convenient geometrical interpretation for a specific application. This will be better illustrated in Section 4.

EXAMPLE 1 In Section 1, we showed that a motion of rotation without translation can be defined directly with respect to the end-effector frame (that is, $\underline{\boldsymbol{x}}_{1}=\underline{\boldsymbol{x}}_{0} \boldsymbol{r}_{E}$ ), but when this motion is specified with respect to the base frame and $\operatorname{Spin}(3) \ltimes \mathbb{R}^{3}$ operations are used, then a transformation must be performed involving terms of the target frame (i.e. $\left.\underline{\boldsymbol{x}}_{1}=\left(\underline{\boldsymbol{x}}_{0} \boldsymbol{r}_{E} \underline{\boldsymbol{x}}_{0}^{*}\right) \underline{\boldsymbol{x}}_{0}\right)$. On the other hand, if $C M I(3)$ operations are used, the specification of this motion is much more straightforward. For instance, let us define a pure rotation with 
respect to the base frame as $\boldsymbol{r}_{B}$. When using $\operatorname{Spin}(3) \ltimes \mathbb{R}^{3}$ operations the final position will be different from the initial position, because for $\underline{\boldsymbol{x}}_{0}=\boldsymbol{r}_{0}+\varepsilon(1 / 2) \boldsymbol{p}_{0} \boldsymbol{r}_{0}$ the new position will be

$$
\underline{\mathcal{T}}\left(\boldsymbol{r}_{B} \underline{x}_{0}\right)=1+\varepsilon \frac{1}{2} \boldsymbol{r}_{B} \boldsymbol{p}_{0} \boldsymbol{r}_{B}^{*}
$$

which is clearly different from the initial position given by $\underline{\mathcal{I}}\left(\underline{\boldsymbol{x}}_{0}\right)=1+\varepsilon(1 / 2) \boldsymbol{p}_{0}$. On the other hand, if the group operation of $C M I(3)$ is used, the motion of rotation without translation can be easily defined directly with respect to the base frame (left action):

$$
\begin{aligned}
\underline{\boldsymbol{x}}_{1} & =\boldsymbol{r}_{B} \otimes \underline{\boldsymbol{x}}_{0}=\underline{\mathcal{T}}\left(\boldsymbol{r}_{B}\right) \underline{\mathcal{T}}\left(\underline{\boldsymbol{x}}_{0}\right) \mathcal{P}\left(\boldsymbol{r}_{B}\right) \mathcal{P}\left(\underline{\boldsymbol{x}}_{0}\right) \\
& =\underline{\mathcal{T}}\left(\underline{\boldsymbol{x}}_{0}\right) \boldsymbol{r}_{B} \boldsymbol{r}_{0} .
\end{aligned}
$$

In this case, the rotation $\boldsymbol{r}_{B}$ is specified with respect to the base frame but applied locally, thus the position does not change; that is, $\mathcal{T}\left(\underline{\boldsymbol{x}}_{1}\right)=\mathcal{T}\left(\underline{\boldsymbol{x}}_{0}\right)$.

\section{Kinematic equation and additional properties of $C M I(3)$}

Given $\underline{x} \in \operatorname{Spin}(3) \ltimes \mathbb{R}^{3}$, the kinematic equation is given by

$$
\underline{\dot{x}}=\frac{1}{2} \underline{\xi} \underline{x},
$$

where $\underline{\dot{x}}$ is the time derivative of $\underline{\boldsymbol{x}}=\boldsymbol{r}+\varepsilon(1 / 2) \boldsymbol{p r}$ (recall that $\boldsymbol{r} \in \operatorname{Spin}(3)$ and $\boldsymbol{p} \in \mathbb{H}_{p}$ ) and $\boldsymbol{\xi}=$ $\boldsymbol{\omega}+\varepsilon(\boldsymbol{v}+\boldsymbol{p} \times \boldsymbol{\omega})$ is the twist expressed in the inertial frame (Wu et al., 2005). In addition, $\boldsymbol{\omega}, \boldsymbol{v} \in \mathbb{H}_{p}$ are pure quaternions that represent the angular and linear velocity, respectively, and $\boldsymbol{p} \times \boldsymbol{\omega}$ is the cross product defined in quaternion form as

$$
p \times \omega \triangleq \frac{p \omega-\omega p}{2} .
$$

Thanks to the isomorphism $\left(\mathbb{R}^{3},+\right) \cong\left(\mathbb{H}_{p},+\right)$, the cross-product (16) between pure quaternions yields the same result of the cross product between the corresponding vectors in $\mathbb{R}^{3}$.

If $\underline{x}$ is the result of the composition of several rigid motions (e.g. the combination of several intermediate rigid motions of a serial kinematic chain), that is $\underline{x}=\underline{x}_{1} \cdots \underline{x}_{n}$, then the resultant twist is the sum of all individual twists expressed in the inertial frame (Adorno, 2011). It is important to recall that, due to the coupling between rotation and translation of $\operatorname{Spin}(3) \ltimes \mathbb{R}^{3}$, the resultant linear velocity is strongly influenced by the angular velocities of intermediate elements of the kinematic chain.

Hence, we seek the twist associated to a composition of rigid motions under the $C M I(3)$ operation, which tends to be much simpler as motions are decoupled. More specifically, we want to find the twist $\underline{\xi}_{d}$ that satisfies

$$
\underline{\dot{x}}=\frac{1}{2} \underline{\xi}_{d} \underline{x}
$$

where $\underline{\boldsymbol{x}}=\underline{\boldsymbol{x}}_{1} \otimes \cdots \otimes \underline{\boldsymbol{x}}_{n}$. Before deriving the final result, first we introduce some auxiliary lemmas. 
LEMMA 6 A sequence of decompositional multiplications always result in a decomposed movement, that is

$$
\underline{\boldsymbol{x}}_{1} \otimes \underline{\boldsymbol{x}}_{2} \otimes \ldots \otimes \underline{\boldsymbol{x}}_{n}=\left(\prod_{l=1}^{n} \underline{\mathcal{T}}\left(\underline{\boldsymbol{x}}_{l}\right)\right)\left(\prod_{l=1}^{n} \mathcal{P}\left(\underline{\boldsymbol{x}}_{l}\right)\right)
$$

Proof. From Lemmas 2 and 3 we obtain

$$
\begin{aligned}
\underline{\boldsymbol{x}}_{1} & \otimes \underline{\boldsymbol{x}}_{2} \otimes \underline{\boldsymbol{x}}_{3} \otimes \ldots \otimes \underline{\boldsymbol{x}}_{n} \\
& =\left\{\left[\left(\underline{\boldsymbol{x}}_{1} \otimes \underline{\boldsymbol{x}}_{2}\right) \otimes \underline{\boldsymbol{x}}_{3}\right] \otimes \ldots\right\} \otimes \underline{\boldsymbol{x}}_{n} \\
& \left.=\left\{\left[\underline{\mathcal{T}} \underline{\boldsymbol{x}}_{1} \otimes \underline{\boldsymbol{x}}_{2}\right) \underline{\mathcal{T}}\left(\underline{\boldsymbol{x}}_{3}\right) \mathcal{P}\left(\underline{\boldsymbol{x}}_{1} \otimes \underline{\boldsymbol{x}}_{2}\right) \mathcal{P}\left(\underline{\boldsymbol{x}}_{3}\right)\right] \otimes \ldots\right\} \otimes \underline{\boldsymbol{x}}_{n} \\
& \vdots \\
& =\underline{\mathcal{T}}\left(\underline{\boldsymbol{x}}_{1}\right) \ldots \underline{\mathcal{T}}\left(\underline{\boldsymbol{x}}_{n}\right) \mathcal{P}\left(\underline{\boldsymbol{x}}_{1}\right) \ldots \mathcal{P}\left(\underline{\boldsymbol{x}}_{n}\right) .
\end{aligned}
$$

Lemma 6 is important because a sequence of transformations under the decompositional multiplication preserves the cross-motion invariance, since the decomposition propagates through the whole transformation.

LEMMA 7 Given $\underline{\boldsymbol{x}} \in \underline{\mathcal{S}}$ and $\boldsymbol{h} \in \mathbb{H}$, then $\underline{\mathcal{T}}(\underline{\boldsymbol{x}})(\varepsilon \boldsymbol{h})=(\varepsilon \boldsymbol{h}) \underline{\mathcal{T}}(\underline{\boldsymbol{x}})=\varepsilon \boldsymbol{h}$.

Proof. Since $\underline{x}$ has unit norm, then $\underline{\mathcal{T}}(\underline{\boldsymbol{x}})=1+\varepsilon(1 / 2) \boldsymbol{p}$ and the result is obtained by direct calculation using the fact that $\varepsilon^{2}=0$.

LEMma 8 Given $\underline{\boldsymbol{x}}_{1}, \ldots, \underline{\boldsymbol{x}}_{n} \in \underline{\mathcal{S}}$

$$
\frac{d}{d t}\left(\prod_{l=1}^{n} \mathcal{P}\left(\underline{\boldsymbol{x}}_{l}\right)\right)=\frac{1}{2}\left(\sum_{m=1}^{n} \boldsymbol{\omega}_{m}^{\prime}\right)\left(\prod_{l=1}^{n} \mathcal{P}\left(\underline{\boldsymbol{x}}_{l}\right)\right),
$$

where $\boldsymbol{\omega}_{m}^{\prime}=\operatorname{Ad}\left(\prod_{l=1}^{m-1} \mathcal{P}\left(\underline{\boldsymbol{x}}_{l}\right)\right) \boldsymbol{\omega}_{m}$ and $\boldsymbol{\omega}_{m} \in \mathbb{H}_{p}$ satisfies $\mathcal{P}\left(\underline{\dot{x}}_{m}\right)=(1 / 2) \boldsymbol{\omega}_{m} \mathcal{P}\left(\underline{\boldsymbol{x}}_{m}\right)$.

Proof. From (15), we see that the kinematic equation of only the primary part is $\mathcal{P}^{\prime}\left(\underline{\boldsymbol{x}}_{l}\right)=\mathcal{P}\left(\underline{\boldsymbol{x}}_{l}\right)=$ $(1 / 2) \boldsymbol{\omega}_{l} \mathcal{P}\left(\underline{\boldsymbol{x}}_{l}\right)$, where $\mathcal{P}^{\prime}\left(\underline{\boldsymbol{x}}_{l}\right) \triangleq \frac{d}{d t} \mathcal{P}\left(\underline{\boldsymbol{x}}_{l}\right)$-this is commonly known as the quaternion propagation equation (Kuipers, 2002). Hence

$$
\begin{aligned}
\frac{d}{d t}\left(\prod_{l=1}^{n} \mathcal{P}\left(\underline{\boldsymbol{x}}_{l}\right)\right) & =\sum_{m=1}^{n}\left(\prod_{l=1}^{m-1} \mathcal{P}\left(\underline{\boldsymbol{x}}_{l}\right)\right) \mathcal{P}^{\prime}\left(\underline{\boldsymbol{x}}_{m}\right)\left(\prod_{l=m+1}^{n} \mathcal{P}\left(\underline{\boldsymbol{x}}_{l}\right)\right) \\
& =\frac{1}{2} \sum_{m=1}^{n}\left(\prod_{l=1}^{m-1} \mathcal{P}\left(\underline{\boldsymbol{x}}_{l}\right)\right) \boldsymbol{\omega}_{m}\left(\prod_{l=m}^{n} \mathcal{P}\left(\underline{\boldsymbol{x}}_{l}\right)\right) .
\end{aligned}
$$


Let $\boldsymbol{\omega}_{m}^{\prime} \triangleq \operatorname{Ad}\left(\prod_{l=1}^{m-1} \mathcal{P}\left(\underline{\boldsymbol{x}}_{l}\right)\right) \boldsymbol{\omega}_{m}$, then

$$
\begin{aligned}
\frac{1}{2} \sum_{m=1}^{n}\left(\prod_{l=1}^{m-1} \mathcal{P}\left(\underline{\boldsymbol{x}}_{l}\right)\right) \boldsymbol{\omega}_{m}\left(\prod_{l=m}^{n} \mathcal{P}\left(\underline{\boldsymbol{x}}_{l}\right)\right) & =\frac{1}{2} \sum_{m=1}^{n} \boldsymbol{\omega}_{m}^{\prime}\left(\prod_{l=1}^{m-1} \mathcal{P}\left(\underline{\boldsymbol{x}}_{l}\right)\right)\left(\prod_{l=m}^{n} \mathcal{P}\left(\underline{\boldsymbol{x}}_{l}\right)\right) \\
& =\frac{1}{2}\left(\sum_{m=1}^{n} \boldsymbol{\omega}_{m}^{\prime}\right)\left(\prod_{l=1}^{n} \mathcal{P}\left(\underline{\boldsymbol{x}}_{l}\right)\right) .
\end{aligned}
$$

THEOREM 2 Given $\underline{\boldsymbol{x}} \in C M I(3)$ such that $\underline{\boldsymbol{x}}=\underline{\boldsymbol{x}}_{1} \otimes \cdots \otimes \underline{\boldsymbol{x}}_{n}$ with $\underline{\boldsymbol{x}}_{l}=\boldsymbol{r}_{l}+\varepsilon(1 / 2) \boldsymbol{p}_{l} \boldsymbol{r}_{l}$, the twist that satisfies the kinematic equation

$$
\underline{\dot{x}}=\frac{1}{2} \underline{\xi_{d}} \underline{x}
$$

is $\underline{\xi}_{d}=\omega_{d}+\varepsilon\left(v_{d}+p_{d} \times \omega_{d}\right)$, where the total linear velocity is $\boldsymbol{v}_{d}=\sum_{l=1}^{n} \boldsymbol{v}_{l}$, the total translation is $\boldsymbol{p}_{d}=\sum_{l=1}^{n} \boldsymbol{p}_{l}$ and the total angular velocity is $\boldsymbol{\omega}_{d}=\sum_{m=1}^{n} \boldsymbol{\omega}_{m}^{\prime}$, where $\boldsymbol{\omega}_{m}^{\prime}=\operatorname{Ad}\left(\prod_{l=1}^{m-1} \mathcal{P}\left(\underline{\boldsymbol{x}}_{l}\right)\right) \boldsymbol{\omega}_{m}$.

Proof. Since $\underline{\boldsymbol{x}}=\underline{\boldsymbol{x}}_{1} \otimes \cdots \otimes \underline{\boldsymbol{x}}_{n}$, then by Lemma 6

$$
\underline{\boldsymbol{x}}=\left(\prod_{l=1}^{n} \underline{\mathcal{T}}\left(\underline{\boldsymbol{x}}_{l}\right)\right)\left(\prod_{l=1}^{n} \mathcal{P}\left(\underline{\boldsymbol{x}}_{l}\right)\right) .
$$

In addition, the time derivative of $\underline{\mathcal{T}}\left(\underline{\boldsymbol{x}}_{l}\right)$-denoted by $\underline{\mathcal{T}} \underline{\boldsymbol{T}}^{\prime}\left(\underline{\boldsymbol{x}}_{l}\right) \triangleq \frac{d}{d t} \underline{\mathcal{T}}\left(\underline{\boldsymbol{x}}_{l}\right)$-is $\underline{\mathcal{T}}\left(\underline{\boldsymbol{x}}_{l}\right)=\varepsilon\left(\boldsymbol{v}_{l} / 2\right)$, where $\boldsymbol{v}_{l} \triangleq \dot{\boldsymbol{p}}_{l}$ so we use Lemmas 7 and 8 to obtain

$$
\underline{\dot{\boldsymbol{x}}}=\left(\sum_{l=1}^{n} \underline{\mathcal{T}}^{\prime}\left(\underline{\boldsymbol{x}}_{l}\right)\right)\left(\prod_{l=1}^{n} \mathcal{P}\left(\underline{\boldsymbol{x}}_{l}\right)\right)+\frac{1}{2}\left(\prod_{l=1}^{n} \underline{\mathcal{T}}\left(\underline{\boldsymbol{x}}_{l}\right)\right)\left(\sum_{m=1}^{n} \boldsymbol{\omega}_{m}^{\prime}\right)\left(\prod_{l=1}^{n} \mathcal{P}\left(\underline{\boldsymbol{x}}_{l}\right)\right),
$$

where $\boldsymbol{\omega}_{m}^{\prime}=\operatorname{Ad}\left(\prod_{l=1}^{m-1} \mathcal{P}\left(\underline{\boldsymbol{x}}_{l}\right)\right) \boldsymbol{\omega}_{m}$. Since $\underline{\mathcal{T}}\left(\underline{\boldsymbol{x}}_{m}\right) \underline{\mathcal{T}}^{\prime}\left(\underline{\boldsymbol{x}}_{l}\right)=\underline{\mathcal{T}}^{\prime}\left(\underline{\boldsymbol{x}}_{l}\right)$ for any $\underline{\boldsymbol{x}}_{m}, \underline{\boldsymbol{x}}_{l}$, hence

$$
\begin{aligned}
\underline{\dot{\boldsymbol{x}}} & =\left(\prod_{l=1}^{n} \underline{\mathcal{T}}\left(\underline{\boldsymbol{x}}_{l}\right)\right)\left[\left(\sum_{l=1}^{n} \underline{\mathcal{T}}^{\prime}\left(\underline{\boldsymbol{x}}_{l}\right)\right)+\frac{1}{2}\left(\sum_{m=1}^{n} \boldsymbol{\omega}_{m}^{\prime}\right)\right]\left(\prod_{l=1}^{n} \mathcal{P}\left(\underline{\boldsymbol{x}}_{l}\right)\right) \\
& =\frac{1}{2}\left(\prod_{l=1}^{n} \underline{\mathcal{T}}\left(\underline{\boldsymbol{x}}_{l}\right)\right)\left[\sum_{m=1}^{n} \boldsymbol{\omega}_{m}^{\prime}+\varepsilon \sum_{l=1}^{n} \boldsymbol{v}_{l}\right]\left(\prod_{l=1}^{n} \mathcal{P}\left(\underline{\boldsymbol{x}}_{l}\right)\right) \\
& =\frac{1}{2}\left(\prod_{l=1}^{n} \underline{\mathcal{T}}\left(\underline{\boldsymbol{x}}_{l}\right)\right)\left[\boldsymbol{\omega}_{d}+\varepsilon \boldsymbol{v}_{d}\right]\left(\prod_{l=1}^{n} \mathcal{P}\left(\underline{\boldsymbol{x}}_{l}\right)\right),
\end{aligned}
$$

where $\boldsymbol{v}_{d} \triangleq \sum_{l=1}^{n} \boldsymbol{v}_{l}$ and $\omega_{d} \triangleq \sum_{m=1}^{n} \omega_{m}^{\prime}$. Let $\underline{\xi}_{d}^{\prime} \triangleq \omega_{d}+\varepsilon \boldsymbol{v}_{d}$ and $\underline{\boldsymbol{\xi}}_{d}=\operatorname{Ad}\left(\prod_{l=1}^{n} \underline{\mathcal{T}}\left(\underline{\boldsymbol{x}}_{l}\right)\right) \underline{\boldsymbol{\xi}}_{d}^{\prime}$, thus

$$
\underline{\dot{x}}=\frac{1}{2} \underline{\boldsymbol{\xi}}_{d}\left(\prod_{l=1}^{n} \underline{\mathcal{T}}\left(\underline{\boldsymbol{x}}_{l}\right)\right)\left(\prod_{l=1}^{n} \mathcal{P}\left(\underline{\boldsymbol{x}}_{l}\right)\right)=\frac{1}{2} \underline{\boldsymbol{\xi}}_{d} \underline{\boldsymbol{x}} .
$$


Let $\boldsymbol{p}_{d}=\sum_{l=1}^{n} \boldsymbol{p}_{l}$ and recall (16) to obtain

$$
\begin{aligned}
\underline{\boldsymbol{\xi}}_{d} & =\operatorname{Ad}\left(\prod_{l=1}^{n} \underline{\mathcal{T}}\left(\underline{\boldsymbol{x}}_{l}\right)\right) \underline{\boldsymbol{\xi}}_{d}^{\prime} \\
& =\left(\prod_{l=1}^{n} \underline{\mathcal{T}}\left(\underline{\boldsymbol{x}}_{l}\right)\right)\left(\boldsymbol{\omega}_{d}+\varepsilon \boldsymbol{v}_{d}\right)\left(\prod_{l=1}^{n} \underline{\mathcal{T}}\left(\underline{\boldsymbol{x}}_{l}\right)\right)^{*} \\
& =\left(1+\varepsilon \sum_{l=1}^{n} \frac{\boldsymbol{p}_{l}}{2}\right)\left(\boldsymbol{\omega}_{d}+\varepsilon \boldsymbol{v}_{d}\right)\left(1-\varepsilon \sum_{l=1}^{n} \frac{\boldsymbol{p}_{l}}{2}\right) \\
& =\boldsymbol{\omega}_{d}+\varepsilon\left(\boldsymbol{v}_{d}+\boldsymbol{p}_{d} \times \boldsymbol{\omega}_{d}\right) .
\end{aligned}
$$

It is important to note that the twist $\underline{\boldsymbol{\xi}}_{d}$ given in Theorem 2 is in fact simpler than the one of (15), since the translational components $\boldsymbol{p}_{d}$ and $\boldsymbol{v}_{d}$ are just the sum of the contributions of any intermediate translations and velocities, respectively, without any influence of the intermediate rotations and angular velocities. This is not the case for $\operatorname{Spin}(3) \ltimes \mathbb{R}^{3}$. The resultant angular velocity, however, is the same for both groups.

\subsection{Additional properties of $C M I(3)$}

LEMMA 9 (COMMUTATIVITY WITH TRANSLATION)

$$
\underline{\mathcal{T}}\left(\underline{\boldsymbol{x}}_{1}\right) \otimes \underline{\boldsymbol{x}}_{2}=\underline{\boldsymbol{x}}_{2} \otimes \underline{\mathcal{T}}\left(\underline{\boldsymbol{x}}_{1}\right)=\underline{\mathcal{T}}\left(\underline{\boldsymbol{x}}_{1}\right) \underline{\boldsymbol{x}}_{2} .
$$

Proof. Since $\underline{\mathcal{T}}\left(\underline{\boldsymbol{x}}_{1}\right) \underline{\mathcal{I}}\left(\underline{\boldsymbol{x}}_{2}\right)=\underline{\mathcal{T}}\left(\underline{\boldsymbol{x}}_{2}\right) \underline{\mathcal{T}}\left(\underline{\boldsymbol{x}}_{1}\right)$ and $\mathcal{P}\left(\underline{\mathcal{I}}\left(\underline{\boldsymbol{x}}_{1}\right)\right)=1$, the result follows from direct calculation.

Proposition 1 The left action $\underline{\boldsymbol{x}}_{a}$ on the target frame $\underline{\boldsymbol{x}}_{t}$, under the decompositional multiplication, is independent of the position of the reference frame.

Proof. Let us consider a left action $\underline{\boldsymbol{x}}_{a}^{\text {ref }}$, specified with respect to a reference frame $\mathcal{F}_{\text {ref }}$, which not necessarily is the inertial frame $\mathcal{F}$. This action will be applied to a target frame $\mathcal{F}_{t}$, represented by $\underline{\boldsymbol{x}}_{t}$, where $\underline{\boldsymbol{x}}_{t}$ is specified with respect to $\mathcal{F}$. The final transformation, with respect to $\mathcal{F}_{\text {ref }}$, is given by

$$
\underline{\boldsymbol{x}}_{f}^{\mathrm{ref}}=\underline{\boldsymbol{x}}_{a}^{\mathrm{ref}} \otimes\left(\underline{\boldsymbol{x}}_{t}^{\mathrm{ref}}\right)
$$

where $\underline{x}_{t}^{\text {ref }}=\underline{\boldsymbol{x}}^{\text {ref }} \underline{\boldsymbol{x}}_{t}$ and $\underline{\boldsymbol{x}}^{\text {ref }}$ is the transformation from $\mathcal{F}_{\text {ref }}$ to $\mathcal{F}$. If the final transformation is to be specified with respect to $\mathcal{F}$, then (18) becomes

$$
\begin{aligned}
\underline{\boldsymbol{x}}_{f} & =\underline{\boldsymbol{x}}^{\mathrm{ref} *}\left(\underline{\boldsymbol{x}}_{a}^{\mathrm{ref}} \otimes \underline{\boldsymbol{x}}_{t}^{\mathrm{ref}}\right) \\
& =\left(\operatorname{Ad}\left(\mathcal{P}\left(\underline{\boldsymbol{x}}^{\mathrm{ref*}}\right)\right) \underline{\boldsymbol{x}}_{a}^{\mathrm{ref}}\right) \otimes \underline{\boldsymbol{x}}_{t},
\end{aligned}
$$

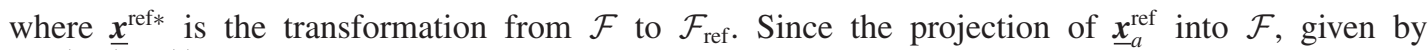
$\operatorname{Ad}\left(\mathcal{P}\left(\underline{\boldsymbol{x}}^{\text {ref* }}\right)\right) \underline{\boldsymbol{x}}_{a}^{\mathrm{ref}}$, does not take into account the position information of $\underline{\boldsymbol{x}}^{\text {ref }}$, we conclude that the 
left action $\underline{\boldsymbol{x}}_{a}$ on the target frame $\underline{\boldsymbol{x}}_{t}$, under the decompositional multiplication, is independent of the position of the reference frame.

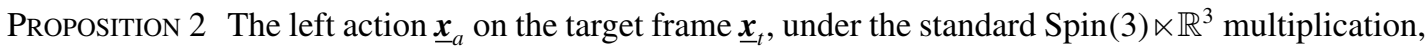
is dependent of the position of the reference frame.

Proof. The proof is similar to the one of Proposition 1. Since $\underline{\boldsymbol{x}}_{f}^{\mathrm{ref}}=\underline{\boldsymbol{x}}_{a}^{\mathrm{ref}} \underline{\boldsymbol{x}}_{t}^{\mathrm{ref}}$, then

$$
\begin{aligned}
\underline{\boldsymbol{x}}_{f} & =\underline{\boldsymbol{x}}^{\mathrm{ref} *} \underline{\boldsymbol{x}}_{a}^{\mathrm{ref}} \underline{\boldsymbol{x}}_{t}^{\mathrm{ref}}=\underline{\boldsymbol{x}}^{\mathrm{ref} *} \underline{\boldsymbol{x}}_{a}^{\mathrm{ref}} \underline{\boldsymbol{x}}^{\mathrm{ref}} \underline{\boldsymbol{x}}_{t} \\
& =\left(\operatorname{Ad}\left(\underline{\boldsymbol{x}}^{\mathrm{ref} *}\right) \underline{\boldsymbol{x}}_{a}^{\mathrm{ref}}\right) \underline{\boldsymbol{x}}_{t} .
\end{aligned}
$$

In this case, since the transformation $\operatorname{Ad}\left(\underline{x}^{\text {ref* }}\right) \underline{x}_{a}^{\text {ref }}$ takes into account both position and orientation information of $\underline{x}^{\text {ref }}$, we conclude that the left action $\underline{\boldsymbol{x}}_{a}$ on the target frame $\underline{\boldsymbol{x}}_{t}$, under the standard $\operatorname{Spin}(3) \ltimes \mathbb{R}^{3}$ multiplication, depends on the position of the reference frame.

The last two propositions show an interesting property of the decompositional multiplication when compared to the standard $\operatorname{Spin}(3) \ltimes \mathbb{R}^{3}$ multiplication. When transforming a frame $\mathcal{F}_{t}$ using $\operatorname{Spin}(3) \ltimes \mathbb{R}^{3}$ operations and specifying a left action $\underline{\boldsymbol{x}}_{a}^{\text {ref }}$ with respect to a reference frame different from the inertial frame $\mathcal{F}$, uncertainties in the position of this reference frame will affect the final transformation. On the other hand, if this left action is applied using the decompositional multiplication, uncertainties in the position of the reference frame will not affect the final transformation; in fact, the position of the reference frame is completely irrelevant.

\subsection{Number of elementary operations involved in the computation of CMI(3) operations}

In this section we present the number of elementary operations (addition and multiplication) involved in the calculation of both the standard multiplication of $\operatorname{Spin}(3) \ltimes \mathbb{R}^{3}$ and the decompositional multiplication of $C M I(3)$.

The cost of multiplying two unit dual quaternions $\underline{x}_{1}$ and $\underline{x}_{2}$ under the standard multiplication is equivalent to the cost of the following operation

$$
\underline{\boldsymbol{x}}_{1} \underline{\boldsymbol{x}}_{2}=\mathcal{P}\left(\underline{\boldsymbol{x}}_{1}\right) \mathcal{P}\left(\underline{\boldsymbol{x}}_{2}\right)+\varepsilon\left(\mathcal{P}\left(\underline{\boldsymbol{x}}_{1}\right) \mathcal{D}\left(\underline{\boldsymbol{x}}_{2}\right)+\mathcal{D}\left(\underline{\boldsymbol{x}}_{1}\right) \mathcal{P}\left(\underline{\boldsymbol{x}}_{2}\right)\right),
$$

where $\mathcal{P}(\cdot)$ and $\mathcal{D}(\cdot)$ are given by (3). Hence, the resulting cost is the cost of three multiplications of quaternions (one multiplication for the primary part and two multiplications for the dual part) and one addition of quaternion for the dual part. According to Adorno (2011), the multiplication of quaternions requires 16 multiplications of real numbers and twelve additions of real numbers, whereas the addition of quaternions requires four additions of real numbers. This way, the total cost of multiplying two dual quaternions under the standard multiplication of $\operatorname{Spin}(3) \ltimes \mathbb{R}^{3}$ is 48 multiplications of real numbers and 40 additions of real numbers.

On the other hand, the cost of multiplying two unit dual quaternions $\underline{\boldsymbol{x}}_{1}$ and $\underline{\boldsymbol{x}}_{2}$ under the decompositional multiplication of $C M I(3)$ corresponds to the cost of the following operation

$$
\begin{aligned}
\underline{\boldsymbol{x}}_{1} \otimes \underline{\boldsymbol{x}}_{2} & =\underline{\mathcal{T}}\left(\underline{\boldsymbol{x}}_{1}\right) \underline{\mathcal{T}}\left(\underline{\boldsymbol{x}}_{2}\right) \mathcal{P}\left(\underline{\boldsymbol{x}}_{1}\right) \mathcal{P}\left(\underline{\boldsymbol{x}}_{2}\right) \\
& =\underline{\boldsymbol{x}}_{1} \mathcal{P}\left(\underline{\boldsymbol{x}}_{1}\right)^{*} \underline{\boldsymbol{x}}_{2} \mathcal{P}\left(\underline{\boldsymbol{x}}_{2}\right)^{*} \mathcal{P}\left(\underline{\boldsymbol{x}}_{1}\right) \mathcal{P}\left(\underline{\boldsymbol{x}}_{2}\right)
\end{aligned}
$$


TABLE 1 Main differences between $\operatorname{Spin}(3) \ltimes \mathbb{R}^{3}$ and CMI(3)

\begin{tabular}{|c|c|}
\hline $\operatorname{Spin}(3) \ltimes \mathbb{R}^{3}$ & $C M I(3)$ \\
\hline $\begin{array}{l}\text { Cross-motion variant (rotation affects } \\
\text { translation) }\end{array}$ & $\begin{array}{l}\text { Cross-motion invariant (rotation does not affect } \\
\text { translation) }\end{array}$ \\
\hline $\begin{array}{l}\text { Translation does not commute with arbitrary } \\
\quad \text { rigid motions: } \underline{\mathcal{I}}\left(\underline{x}_{1}\right) \underline{x}_{2} \neq \underline{x}_{2} \underline{\mathcal{I}}\left(\underline{x}_{1}\right)\end{array}$ & $\begin{array}{l}\text { Translation commutes with arbitrary rigid } \\
\text { motions: } \underline{\mathcal{I}}\left(\underline{\boldsymbol{x}}_{1}\right) \otimes \underline{\boldsymbol{x}}_{2}=\underline{\boldsymbol{x}}_{2} \otimes \underline{\mathcal{I}}\left(\underline{\boldsymbol{x}}_{1}\right)\end{array}$ \\
\hline $\begin{array}{l}\text { Transformation depends on the position of the } \\
\text { reference frame }\end{array}$ & $\begin{array}{l}\text { Transformation does not depend on the position } \\
\text { of the reference frame }\end{array}$ \\
\hline $\begin{array}{l}\text { Does not preserve the direction of transformed } \\
\text { trajectories }\end{array}$ & $\begin{array}{l}\text { Preserves the direction of transformed } \\
\text { trajectories }\end{array}$ \\
\hline $\begin{array}{l}\text { More complex kinematic equations for a } \\
\text { sequence of rigid motions }\end{array}$ & $\begin{array}{l}\text { Less complex kinematic equations for a } \\
\text { sequence of rigid motions }\end{array}$ \\
\hline $\begin{array}{l}\text { Standard multiplication requires } 48 \\
\text { multiplications of real numbers and } 40 \\
\text { additions of real numbers }\end{array}$ & $\begin{array}{l}\text { Decompositional multiplication requires } 67 \\
\text { multiplications of real numbers and } 52 \\
\text { additions of real numbers }\end{array}$ \\
\hline
\end{tabular}

$$
\begin{aligned}
& =\left[\mathcal{P}\left(\underline{\boldsymbol{x}}_{1}\right)+\varepsilon \mathcal{D}\left(\underline{\boldsymbol{x}}_{1}\right)\right] \mathcal{P}\left(\underline{\boldsymbol{x}}_{1}\right)^{*}\left[\mathcal{P}\left(\underline{\boldsymbol{x}}_{2}\right)+\varepsilon \mathcal{D}\left(\underline{\boldsymbol{x}}_{2}\right)\right] \mathcal{P}\left(\underline{\boldsymbol{x}}_{2}\right)^{*} \mathcal{P}\left(\underline{\boldsymbol{x}}_{1}\right) \mathcal{P}\left(\underline{\boldsymbol{x}}_{2}\right) \\
& =\mathcal{P}\left(\underline{\boldsymbol{x}}_{1}\right) \mathcal{P}\left(\underline{\boldsymbol{x}}_{2}\right)+\varepsilon\left[\mathcal{D}\left(\underline{\boldsymbol{x}}_{2}\right) \mathcal{P}\left(\underline{\boldsymbol{x}}_{2}\right)^{*} \mathcal{P}\left(\underline{\boldsymbol{x}}_{1}\right)+\mathcal{D}\left(\underline{\boldsymbol{x}}_{1}\right)\right] \mathcal{P}\left(\underline{\boldsymbol{x}}_{2}\right),
\end{aligned}
$$

which requires four multiplications of quaternions (one multiplication for the primary part and three multiplications for the dual part), one addition of quaternion and one conjugation of quaternion. Thus, the total cost of multiplying two unit quaternions under the decompositional multiplication of $C M I(3)$ is 67 multiplications of real numbers (the conjugation of a quaternion requires three multiplications of real numbers) and 52 additions of real numbers. These results are summarized in Table 1.

\section{Numerical examples}

In this first example, consider a trajectory expressed in the inertial frame $\mathcal{F}$, given by $\underline{\boldsymbol{h}}(t)=\boldsymbol{r}(t)+$ $\varepsilon(1 / 2) \boldsymbol{p}(t) \boldsymbol{r}(t)$, where $\boldsymbol{p}=\hat{\imath} \cos t+\hat{\jmath} \sin t+\hat{k} t$ and $\boldsymbol{r}=\cos (t / 2)+\hat{\imath} \sin (t / 2)$. The goal is to apply the transformation $\underline{\boldsymbol{h}}_{a}^{\text {ref }}$, where $\boldsymbol{p}_{a}^{\text {ref }}=\hat{\imath}+\hat{\jmath}$ and $\boldsymbol{r}_{a}^{\text {ref }}=\cos (\pi / 4)+\hat{\jmath} \sin (\pi / 4)$, to the trajectory $\underline{\boldsymbol{h}}(t)$ using the frame $\mathcal{F}_{\text {ref }}$ as the reference for the action, where $\boldsymbol{p}_{\text {ref }}=2.5 \hat{\imath}+\hat{\jmath}+3.5 \hat{k}$ and $\boldsymbol{r}_{\text {ref }}=\cos (\pi / 2)+\hat{k} \sin (\pi / 2)$. In $C M I(3)$, when the transformation is specified with respect to $\mathcal{F}_{\text {ref }}$, but the trajectory is expressed in the inertial frame $\mathcal{F}$, the transformed trajectory is given by $\underline{\boldsymbol{g}}(t)=\left(\operatorname{Ad}\left(\mathcal{P}\left(\underline{\boldsymbol{h}}_{\mathrm{ref}}\right)\right) \underline{\boldsymbol{h}}_{a}^{\mathrm{ref}}\right) \otimes \underline{\boldsymbol{h}}(t)$, where $\underline{\boldsymbol{h}}_{\text {ref }}$ provides the rigid transformation from $\mathcal{F}$ to $\mathcal{F}_{\text {ref }}$ (see (19) of Proposition 1). When the transformation $\underline{\boldsymbol{h}}_{a}^{\text {ref }}$ belongs to $\operatorname{Spin}(3) \ltimes \mathbb{R}^{3}$ and is specified with respect to $\mathcal{F}_{\text {ref }}$, but the trajectory is expressed in $\mathcal{F}$, the transformed trajectory is given by $\underline{\boldsymbol{f}}_{(t)}=\left(\operatorname{Ad}\left(\underline{\boldsymbol{h}}_{\mathrm{ref}}\right) \underline{\boldsymbol{h}}_{a}^{\mathrm{ref}}\right) \underline{\boldsymbol{h}}(t)$ - see (20) of Proposition 2. Note that, although the action $\underline{\boldsymbol{h}}_{a}^{\text {ref }}$ is specified with respect to $\mathcal{F}_{\text {ref }}$, both original and modified trajectories are specified with respect to the fixed frame. The original trajectory $\underline{\boldsymbol{h}}(t)$ is represented by a black-dotted curve in Fig. 4, whereas $\boldsymbol{g}(t)$ is represented by a solid-green trajectory and $\boldsymbol{f}(t)$ is represented by a dashedred curve. Because the group operation used to generate $g(t)$ belongs to the cross motion invariant group, translations do not affect rotations and vice-versa. This way, both $\underline{\boldsymbol{h}}(t)$ and $\boldsymbol{g}(t)$ go upward independently of the orientation and the decompositional multiplication preserves the direction of the trajectory. On 


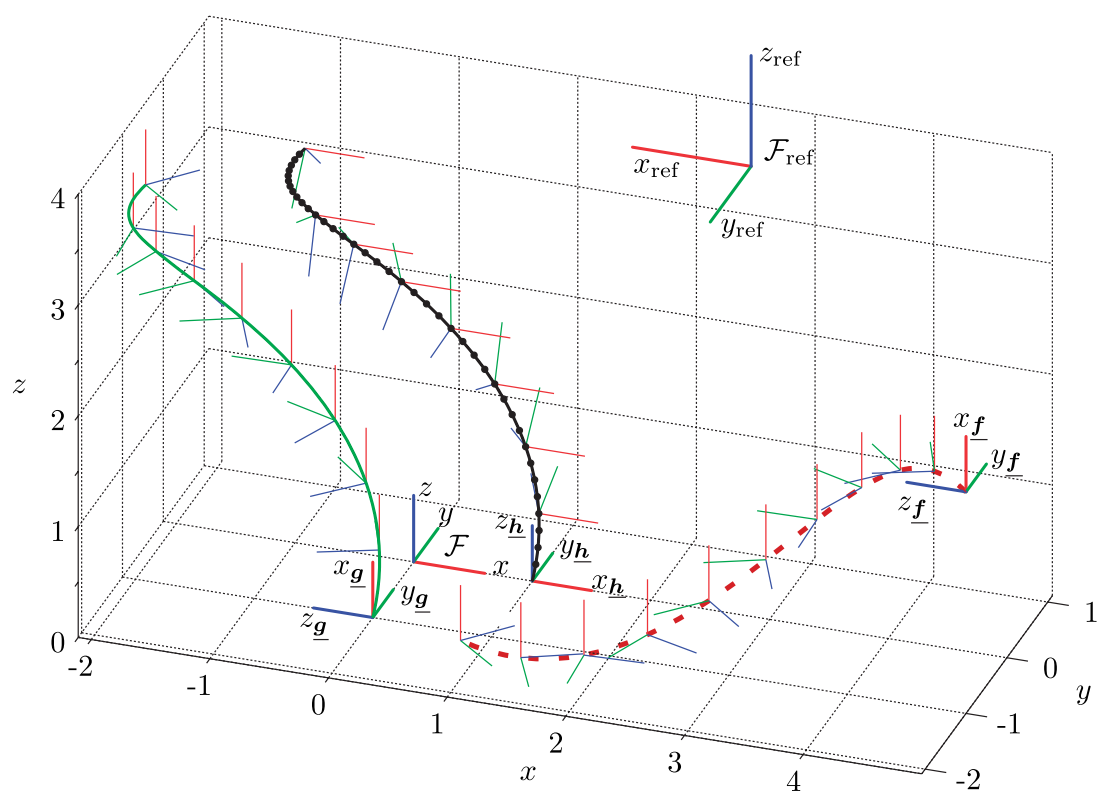

FIG. 4. Original trajectory $\underline{\boldsymbol{h}}(t)$ (black dotted), modified trajectory $\underline{\boldsymbol{g}}(t)$ using $C M I(3)$ transformation (solid green) and modified trajectory $\boldsymbol{f}(t)$ using $\operatorname{Spin}(3) \ltimes \mathbb{R}^{3}$ transformation (dashed red). $\overline{\mathcal{F}}$ is the fixed frame and $\mathcal{F}_{\text {ref }}$ is the reference frame for the transformation. Observe that $C M I(3)$ transformation preserves the direction of the trajectory whereas $\operatorname{Spin}(3) \ltimes \mathbb{R}^{3} \operatorname{transformation}$ does not preserve the direction.

the other hand, $\boldsymbol{f}(t)$ is transformed using $\operatorname{Spin}(3) \ltimes \mathbb{R}^{3}$ operations and thus the dashed-red curve is rotated with respect to the original trajectory, hence the final trajectory has a different direction from the original one.

In this second example, consider a 7-DOF robot manipulator and the associated coordinate systems as shown in Fig. 5, where $\mathcal{F}$ and $\mathcal{F}_{e}$ are the inertial and end-effector frames, respectively. Considering the initial end-effector pose given by the unit dual quaternion $\underline{\boldsymbol{x}}_{e}$, suppose that we want to define a desired rigid motion using the inertial frame $\mathcal{F}$ as the reference frame. This rigid motion consists of a displacement of $0.1 \mathrm{~m}$ in the positive direction of the $x$ axis and $0.3 \mathrm{~m}$ in the negative direction of the $y$ axis - that is, $\boldsymbol{p}=0.1 \hat{\imath}-0.3 \hat{\jmath}$. In addition, we want the end effector to be locally rotated of $-\pi / 2 \mathrm{rad}$ around the $z$ axis-that is, $\boldsymbol{r}=\cos (-\pi / 4)+\hat{k} \sin (-\pi / 4)$. It means that we want to rotate the end effector by using the $z$ axis of a frame that is aligned with the inertial frame but whose origin coincides with the origin of the end-effector frame. The desired rigid motion is given by $\underline{x}_{a}=\boldsymbol{r}+\varepsilon(1 / 2) \boldsymbol{p r}$ and, since we want the rotation to be performed locally (that is, decoupled from the translation), the decompositional multiplication is very convenient, as the final desired pose is given by

$$
\underline{\boldsymbol{x}}_{d}=\underline{\boldsymbol{x}}_{a} \otimes \underline{\boldsymbol{x}}_{e}
$$

and is represented as frame $\mathcal{F}_{d}$ in Fig. 5. Note that, because rotation and translation are decoupled, we can define them separately without worrying about translations being affected by rotations. 
(a)

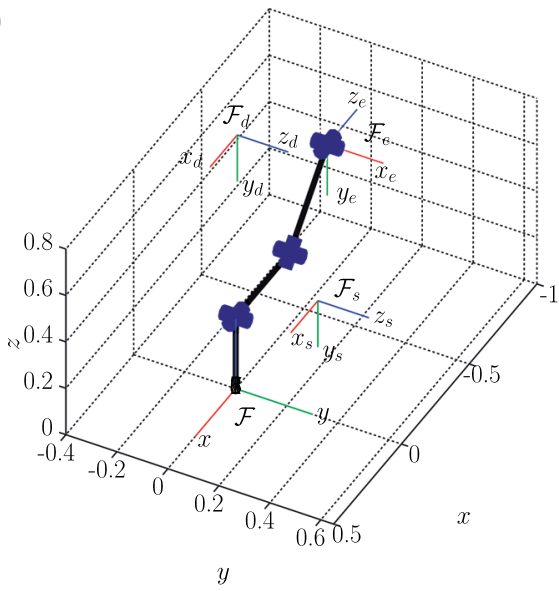

Initial configuration.

(c)

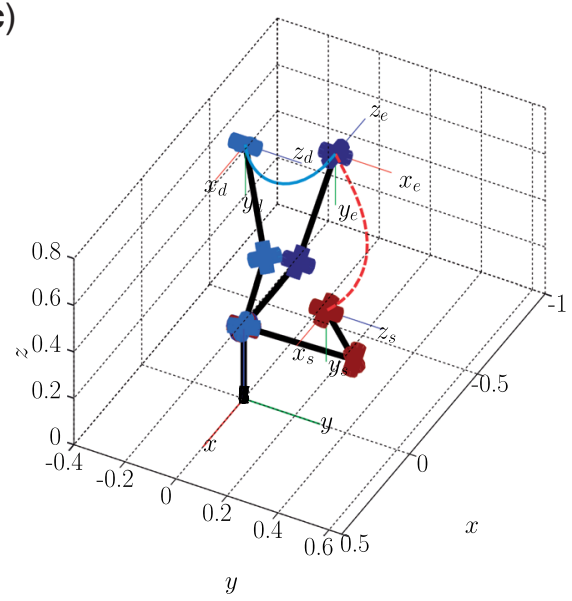

From left to right, the light blue robot corresponds to the final configuration when the desired end-effector pose is given by the decompositional multiplication; the dark blue robot corresponds to the initial configuration; the red robot corresponds to the final configuration when the desired end-effector pose is given by the standard multiplication. (b)

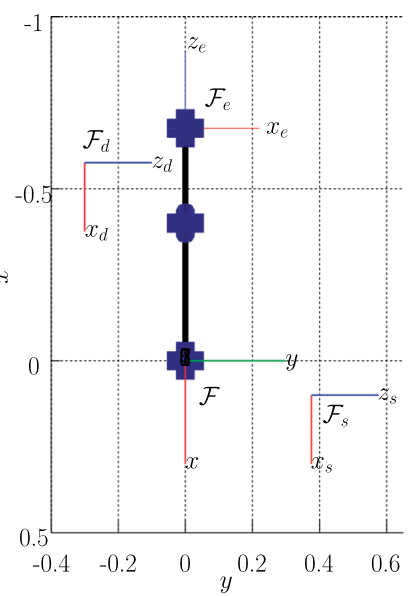

Initial configuration (top view).

(d)

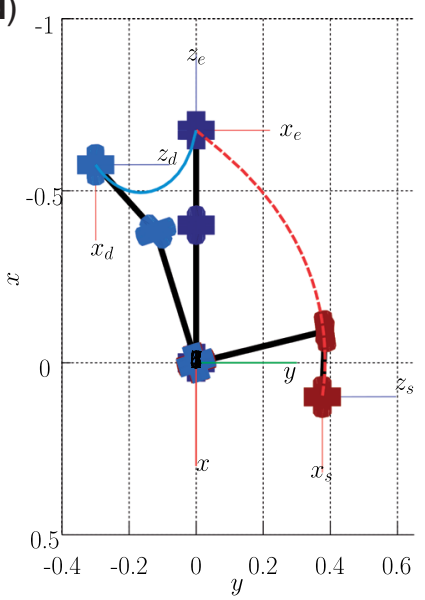

Final configurations (top view). The solid light blue line is the trajectory of the end-effector when the reference is obtained by using the decompositional multiplication, whereas the dashed-red line corresponds to the trajectory of the end effector when the reference is obtained by using the standard multiplication.

FIG. 5. Example of different motions when performing $C M I(3)$ and $\operatorname{Spin}(3) \ltimes \mathbb{R}^{3}$ operations. $\mathcal{F}$ and $\mathcal{F}_{e}$ are the inertial and endeffector frames, respectively, whereas $\mathcal{F}_{d}$ is the final frame obtained by using the $C M I(3)$ decompositional multiplication and $\mathcal{F}_{s}$ is the final frame obtained by using the $\operatorname{Spin}(3) \propto \mathbb{R}^{3}$ standard multiplication.

On the other hand, if we consider $\underline{\boldsymbol{x}}_{a}$ to perform the standard multiplication, the final pose is given by

$$
\underline{\boldsymbol{x}}_{s}=\underline{\boldsymbol{x}}_{a} \underline{\boldsymbol{x}}_{e}
$$


and is represented as frame $\mathcal{F}_{s}$ in Fig. 5. Since the standard multiplication performs a coupled motion, we see that the final rotation is the same, but the final translation is not the intended one. Of course, it is possible to find an equivalent transformation $\underline{y}$ such that $\underline{y}_{e}=\underline{\boldsymbol{x}}_{a} \otimes \underline{\boldsymbol{x}}_{e}$ as shown in Remark 2. However, the advantage of using the decompositional multiplication is that it is usually much more intuitive to think about a decoupled motion to define the desired pose for the end effector.

\section{Conclusions}

This article presented the group $C M I(3)$ and its mathematical properties, as well as some examples in order to show its application to kinematics and to the study of rigid motions in general. Also a thorough and formal comparison with the group $\operatorname{Spin}(3) \ltimes \mathbb{R}^{3}$ has been presented, as summarized in Table 1. Because both $C M I(3)$ and $\operatorname{Spin}(3) \ltimes \mathbb{R}^{3}$ operate on the same underlying structure-the unit dual quaternions - the idea is to use both in a complementary way, leading to a richer algebra of rigid motions. For instance, $C M I(3)$ operations can be, in some cases, more convenient than $\operatorname{Spin}(3) \ltimes \mathbb{R}^{3}$ operations. Because $C M I(3)$ group operation guarantees cross-motion invariance such that rotations and translations do not affect each other, these motions can be specified separately. On the other hand, in some cases the goal is to really have the position affected by the rotation movement; in those cases, $\operatorname{Spin}(3) \ltimes \mathbb{R}^{3}$ operations would be more appropriate.

The kinematic equation was derived for a sequence of rigid motions under the decompositional multiplication. The resultant twist is in fact simpler for $C M I(3)$ than for $\operatorname{Spin}(3) \ltimes \mathbb{R}^{3}$, since in $C M I(3)$ the translational components are just the sum of the contributions of any intermediate translations and linear velocities, without any influence of the intermediate rotations and angular velocities, respectively. This is not the case for $\operatorname{Spin}(3) \ltimes \mathbb{R}^{3}$. The resultant angular velocity, however, is the same for both groups.

Lastly, although the goal of the article was to formally present the mathematical description of the CMI group and only few illustrative examples were shown, this group and its underlying group operation - the decompositional multiplication-can be quite useful in practice. For instance, our implementation of the decompositional multiplication, both in Matlab and $\mathrm{C} / \mathrm{C}++$ (see dqrobotics.sourceforge.net, last accessed on May 10, 2016), was already used in the context of humanrobot interaction and bimanual manipulation in order to simplify the definition of tasks (Adorno et al., 2011).

\section{Funding}

Agence Nationale de la Recherche (ANR) (ANR 07-ROBO-01); Fundação de Amparo à Pesquisa do Estado de Minas Gerais (FAPEMIG) (APQ-00967-14) and Conselho Nacional de Desenvolvimento Científico e Tecnológico (CNPq) (456826/2013-0).

\section{Acknowledgements}

The authors would like to thank Prof. B. Siciliano, Prof. M. Uchiyama and Prof. AP Murray for their helpful remarks regarding the decompositional multiplication.

\section{REFERENCES}

Adorno, B. V. (2011) Two-arm Manipulation: From Manipulators to Enhanced Human-Robot Collaboration [Contribution à la manipulation à deux bras : des manipulateurs à la collaboration homme-robot]. Ph.D. dissertation, Université Montpellier 2, France. 
Adorno, B. V., Bó, A. P. L., Fraisse, P. \& Poignet, P. (2011) Towards a cooperative framework for interactive manipulation involving a human and a humanoid. Proceedings of the IEEE International Conference on Robotics and Automation. China: IEEE, pp. 3777-3783.

Adorno, B. V., Fraisse, P. \& DruOn, S. (2010) Dual position control strategies using the cooperative dual taskspace framework. 2010 IEEE/RSJ International Conference on Intelligent Robots and Systems Taipei: IEEE, pp. 3955-3960.

Craig, J. J. (2005) Introduction To Robotics, Mechanics and Control, 3rd ed. London: Pearson.

DANIILIDIS, K. (1999) Hand-eye calibration using dual quaternions. Int. J. Robotics Res., 18, $286-298$.

KuIPERS, J. (2002) Quaternions and Rotation Sequences: A Primer with Applications to Orbits, Aerospace, and Virtual Reality. New Jersey: Princeton University Press.

LANA, E. P., AdORnO, B. V. \& MAIA, C. A. (2015) A new algebraic approach for the description of robotic manipulation Tasks. 2015 IEEE International Conference on Robotics and Automation (ICRA) Seattle, Washington: IEEE, pp. 3083-3088.

MCCARTHY, J. (1990) Introduction to Theoretical Kinematics, 1st ed. Cambridge, MA: The MIT Press.

Murray, R., LI, Z. \& SASTRY, S. (1994) A Mathematical Introduction to Robotic Manipulation. New York: CRC.

Radavelli, L. A., Pieri, E. R. D., Martins, D. \& Simoni, R. (2014) Points, lines, screws and planes in dual quaternions kinematics. Advances in Robot Kinematics (J. Lenarčič \& O. Khatib eds.) Cham: Springer International Publishing, pp. 285-293.

SELIG, J. M. (2005) Geometric Fundamentals of Robotic, 2nd ed. New York: Springer.

Siciliano, B. \& KHATiB, O. (2008) Springer Handbook of Robotics. New York: Springer.

Siciliano, B., Sciavicco, L., Villani, L. \& Oriolo, G. (2009) Robotics: Modelling, Planning and Control. Advanced Textbooks in Control and Signal Processing. London: Springer-Verlag London.

Spong, M., Hutchinson, S. \& Vidyasagar, M. (2006) Robot Modeling and Control. New Jersey: John Wiley \& Sons.

Wu, Y., Hu, X., Hu, D., LI, T. \& LiAn, J. (2005) Strapdown inertial navigation system algorithms based on dual quaternions. IEEE Trans. Aerosp. Electron. Sys., 41, 110-132. 